$10-1-2002$

\title{
Static Hedging of Standard Options
}

PETER CARR

New York University

LIUREN WU

Fordham University

Follow this and additional works at: https://fordham.bepress.com/crif_working_papers

Part of the Finance and Financial Management Commons

\section{Recommended Citation}

CARR, PETER and WU, LIUREN, "Static Hedging of Standard Options" (2002). CRIF Working Paper series. 22. https://fordham.bepress.com/crif_working_papers/22

This Article is brought to you for free and open access by the Frank J. Petrilli Center for Research in International Finance at DigitalResearch@Fordham. It has been accepted for inclusion in CRIF Working Paper series by an authorized administrator of

DigitalResearch@Fordham. For more information, please contact considine@fordham.edu. 


\title{
Static Hedging of Standard Options*
}

\author{
PETER CARR ${ }^{\dagger}$ \\ Courant Institute, New York University \\ LIUREN WU $\mathrm{U}^{\ddagger}$ \\ Graduate School of Business, Fordham University
}

First draft: July 26, 2002

This version: October 1, 2002

Filename: statichedge10.tex

\footnotetext{
*This version is very preliminary. We welcome comments, especially references to related papers we have inadvertently overlooked. We thank David Hait and OptionMetrics for providing the option data and Alex Mayus for clarifying trading practices. We also thank the participants of the finance workshop at Vanderbilt University. We assume full responsibility for any errors.

$\dagger 251$ Mercer Street, New York, NY 10012; tel: (212) 260-3765; pcarrenyc.rr.com; www. math.nyu.edu/research/carrp/papers.

$\$ 113$ West 60th Street, Suite 616, New York, NY 10023; tel: (212) 636-6117; fax: (212) 765-5573; wu@fordham.edu; www. bnet. fordham. edu/lwu.
} 


\title{
Static Hedging of Standard Options
}

\begin{abstract}
We consider the hedging of derivative securities when the price movement of the underlying asset can exhibit random jumps. Under a one factor Markovian setting, we derive a spanning relation between a long term option and a continuum of short term options. We then apply this spanning relation to the static hedging of long term options with a finite choice of short term, more liquid options based on a quadrature rule. We use Monte Carlo simulation to determine the hedging error introduced by the quadrature approximation and compare this hedging error to the hedging error from a delta hedging strategy based on daily rebalancing in the underlying futures. The simulation results indicate that the two types of strategies have comparable hedging effectiveness in the classic Black-Scholes environment, but that our static hedging strategy strongly outperforms the dynamic delta-hedging strategy when the underlying asset price movement is governed by Merton (1976)'s jump diffusion model. Further simulation exercises indicate that these results are robust to model misspecification, so long as one performs ad hoc adjustments based on the observed implied volatility.

We also compare the hedging effectiveness of the two types of strategies using more than six years of data on S\&P 500 index options. We find that a static hedge using just five call options outperforms daily rebalancing on the delta hedging with the underlying futures. The consistency of this result with our jump model simulations lends empirical support for the existence of jumps of random size in the movement of the S\&P 500 index. We also find that our static strategy performs best when the maturity of the options in the hedging portfolio is close to the maturity of the target option being hedged. As the maturity gap increases, the hedging performance deteriorates moderately, indicating the likely existence of additional random factors such as stochastic volatility.
\end{abstract}

JEL Classification Codes: G12, G13, C52.

KEY WORDS: Jumps; option pricing; static hedging; Monte Carlo; S\&P 500 index options; stochastic volatility. 


\section{Static Hedging of Standard Options}

Over the past two decades, the derivatives market has been expanding dramatically. Accompanying this expansion is an increased urgency in understanding and effectively managing the risks associated with derivative securities. In an ideal setting where the price of the underlying security moves continuously (such as in a diffusion) or with fixed size steps (such as in a binomial tree), derivatives pricing theory provides a framework in which the risks inherent in a derivatives position can be eliminated via dynamic trading in only a small number of securities. In reality, however, large and random price movements happen much more frequently than assumed in the above ideal settings. During the last two decades, we have repeatedly witnessed turmoil in the financial markets such as the 1987 stock market crash, the 1997 Asian crisis, the 1998 Russian default and the ensuing hedge fund crisis, and the tragic events of September 11, 2001. Juxtaposed between these large crises are many more mini-crises, in which prices move sufficiently fast so as to trigger circuit breakers and trading halts. When these crises occur, a dynamic hedging strategy based on small or fixed size movements often breaks down. Worse yet, strategies which involve dynamic hedging in the underlying asset tend to fail precisely when liq-

uidity dries up or when the market makes large moves in either direction. Unfortunately, it is during financial crises such as liquidity gaps or market crashes that effective hedging is most dearly needed. Indeed, several prominent critics have gone further and blamed the emergence of some financial crises on the pursuit of dynamic hedging strategies.

Under a fairly general one-factor Markovian setting, where the market price of a security is allowed not only to move diffusively but also to jump randomly to any non-negative value, this article derives a spanning relation between the future value of a long term European option and the payoff from a continuum of shorter term European options. The required position in each of the shorter term options is proportional to the gamma (second price derivative) that the target option will have when the options in the hedge portfolio expire. Given this spanning result, no arbitrage implies that the target option and the replicating portfolio have the same value for all times until the shorter term options expire. As a result, a long term option can be theoretically hedged, even though large random jumps are allowed in the security price movement. Furthermore, given the static nature of the strategy, one does not need 
to rebalance the hedge portfolio until the shorter term options mature and hence one does not need to worry about market shutdowns and liquidity gaps in the intervening period. Therefore, the strategy remains viable and can become even more useful when the market is in stress.

As transactions costs and illiquidity render the formation of a portfolio with a continuum of options physically impossible, we develop an approximation for the static hedge using only a finite number of options. In particular, we choose the strike levels and the associated portfolio weights based on a Gauss Hermite quadrature method. We use Monte Carlo simulation to gauge the magnitude and distributional characteristics of the hedging error introduced by the quadrature approximation. We compare this hedging error to the hedging error from a delta hedging strategy based on daily rebalancing with the underlying futures. The simulation results indicate that the two strategies have comparable hedging effectiveness in the classic Black-Scholes environment. The mean absolute hedging errors from the two strategies are comparable when the same number of transactions are involved. Nevertheless, since the bid-ask spread is typically lower for the underlying asset than it is for any option, these results favor delta-hedging.

However, this conclusion changes when the simulation is performed under the Merton (1976) jumpdiffusion environment, where the underlying asset price can exhibit discontinuous price movements of random size. We find that the performance of daily delta hedging deteriorates dramatically, while the performance of the static strategy hardly varies. As a result, under the Merton model, a static strategy with merely three options outperforms delta hedging with daily updating. Further simulations indicate that these results are robust to model misspecification, so long as one performs ad hoc adjustments based on the observed implied volatility. Finally, we also find that increasing the rebalancing frequency in the delta hedging strategy cannot revive its performance as long as the underlying price process is allowed to jump randomly. We hence conclude from our simulations that the out-performance of our static hedging strategy over daily delta-hedging when jumps are possible is not due to model misspecification, nor is it due to the approximation error introduced via discrete rebalancing. Rather, this outperformance is due to the fact that the dynamic delta strategy is inherently incapable of dealing with random jumps in the underlying asset price. 
The static hedging strategy is derived in a one factor Markovian setting. The simulation exercises are also performed in this type of environment. To determine whether the superiority of the static hedge in the jump model simulations is robust with respect to possible relaxation of the Markovian assumption, we also compare the performance of the two types of hedging strategies using more than six years of data on S\&P 500 index options. We find that in all the cases considered, a static hedge using as few as five options outperforms daily delta hedging with the underlying futures. The consistency of this result with our jump model simulations lends empirical support for the existence of jumps of random size in the movement of the S\&P 500 index.

We also find that our static strategy performs best when the maturity of the options in the hedging portfolio is close to the maturity of the target option being hedged. As the maturity gap increases, the hedging performance deteriorates moderately, indicating the likely existence of additional random factors such as stochastic volatility. Finally, we look into the sample periods when each type of hedging exercises delivers the worst performance and find that the worst failures occur for the delta hedge when there are large market moves such as during the October 1997 Asian crisis. In contrast, the worst failures for the static hedge are not linked with any identifiable market crises. Hence, investors who wish to avoid experiencing pronounced variability in profit and loss during crises is better off pursuing a static hedge.

The approach of forming a static hedge with a continuum of options is still in its infancy both theoretically and empirically. An interesting facet of this approach is that the very existence of a static hedge and the exact composition of the portfolio relies heavily on the nature of the contract being hedged. For example, consider the seminal results of Breeden and Litzenberger (1978), as foreshadowed in the work of Ross (1976). The elaborations of these results by Green and Jarrow (1987) and Nachman (1988) show how a continuum of options with a common maturity can be used to provide a static hedge, provided that the target payoff depends only upon the price of a single asset at this common maturity. However, if, as in our case, the payoff of this target option is that of a European option, the continuum of options degenerates into the target option itself and hence the spanning relation proposed in Breeden and Litzenberger (1978) degenerates into a tautology. By applying more constraints 
on the underlying process, e.g. a one-factor Markovian process, we are able to span the value of a target European option by a continuum of European options which mature at an earlier date and are thus also potentially more liquid.

As another example on static hedging, Carr, Ellis, and Gupta (1998) show that barrier options can be hedged by semi-static option trading in the Black model and certain mild generalizations. Also related to these results are more recent works by Britten-Jones and Neuberger (2000) and Hodges and Neuberger (2002). The former paper show how a static position in European options can be combined with dynamic trading in their underlying to trade variance. The latter paper shows how the payoffs to various barrier options can be bounded by semi-static trading in their underlying asset. While barrier options represent the most common form of exotic options, there is far greater volume in standard options. Thus, our work focuses on the hedging of the simpler standard European options, but under a general environment where the underlying security price can both move diffusively and jump randomly.

The remainder of this paper is organized as follows. Section I develops the theoretical results underlying our static hedging strategy. Section II uses Monte Carlo simulation to enact a wide variety of scenarios under which the market not only moves diffusively, but also jumps randomly. Under each scenario, we analyze the hedging performance of our static strategy and compare it with dynamic hedging. Section III applies both strategies to the S\&P 500 index options data. Section IV concludes.

\section{Spanning Options with Options}

We develop our main theoretical results in this section. In particular, working in a single factor Markovian setting, we show how the risk of a European option can be spanned by holding a continuum of shorter term European options. We then illustrate how a quadrature rule can be used to approximate the static hedging portfolio with a finite number of nearer term options. 


\section{A. Assumptions and Notation}

We assume frictionless markets and no arbitrage. To fix notation, let $S_{t}$ denote the spot price of an asset (say, a stock or stock index) at time $t \in[0, \mathcal{T}]$, where $\mathcal{T}$ is some arbitrarily distant horizon. For simplicity, we assume that the continuously compounded riskfree rate $r$ and dividend yield $q$ are constant. No arbitrage implies that there exists a risk-neutral probability measure $\mathbb{Q}$ defined on a probability space $(\Omega, \mathcal{F}, \mathbb{Q})$ such that the instantaneous expected rate of return on every asset equals this instantaneous riskfree rate $r$. We restrict our analysis to the class of models in which the risk-neutral evolution of the stock price is Markov in the stock price $S$ and time $t$. Our class of models includes local volatility models, e.g., Dupire (1994), and models based on Lévy processes, e.g., Merton (1976), Madan, Carr, and Chang (1998), and Carr and Wu (2002), but does not include stochastic volatility models such as Heston (1993).

Consider the time- $t$ price of a European call with strike $K$ and maturity $T$, denoted as $C_{t}(K, T)$. Our assumption that the state is fully described by the stock price and time implies that there exists a call pricing function $C(S, t ; K, T ; \Theta)$ such that:

$$
C_{t}(K, T)=C\left(S_{t}, t ; K, T ; \Theta\right), \quad t \in[0, T], K \geq 0, T \in[t, \mathcal{T}]
$$

Thus, the call pricing function relates the call price at $t$ to the state variables $\left(S_{t}, t\right)$, the contractual parameters $(K, T)$, and to a vector of deterministic model parameters $\Theta$.

Finally, let $q(S, t ; K, T ; \Theta)$ denote the probability density function of the asset price under the riskneutral measure $\mathbb{Q}$. This function yields the probability density evaluated at the future price level $K$ and the future time $T$, conditional on the stock price starting at level $S$ at some earlier time $t$. Breeden and Litzenberger (1978) show that this risk-neutral density is related to the second strike derivative of the call pricing function by

$$
q(S, t ; K, T ; \Theta)=e^{-r(T-t)} \frac{\partial^{2} C}{\partial K^{2}}(S, t ; K, T ; \Theta) .
$$




\section{B. Spanning Standard European Options with Shorter Term European Options}

The main theoretical result of the paper comes from the following theorem, which introduces a new spanning relation between the price of an option at one maturity and a continuum of option prices at some nearer maturity. The practical implication of this theorem is that it indicates how one can span the risk of a given option by taking a static position in a continuum of shorter term, usually more liquid, options.

Theorem 1 Under no arbitrage and the Markovian assumption in (1), the time-t value of a European call option maturing at a fixed time $T \geq t$ is related to the time-t values of a continuum of European call options at a shorter maturity $u \in[t, T]$ by,

$$
C(S, t ; K, T ; \Theta)=\int_{0}^{\infty} w(\mathcal{K}) C(S, t ; \mathcal{K}, u ; \Theta) d \mathcal{K}, \quad u \in[t, T]
$$

for all possible nonnegative values of $S$ and time $t \leq u$. The density function $w(\mathcal{K})$ is given by

$$
w(\mathcal{K})=\frac{\partial^{2}}{\partial \mathcal{K}^{2}} C(\mathcal{K}, u ; K, T ; \Theta) .
$$

Note that the spanning relation holds for all possible values of the spot price $S$ and at all times up to the expiry of the options in the spanning portfolio. However, the option weights $w(\mathcal{K})$ are independent of $S$ and $t$. This property dictates the static nature of the spanning relation. Under no arbitrage, once the spanning portfolio is formed, no rebalancing is necessary up until the expiring time $(u)$ of the options in the spanning portfolio. Also note that the weight $w(\mathcal{K})$ on a call of maturity $u$ and strike $\mathcal{K}$ is proportional to the gamma that the target call option will have at time $u$, should the underlying be at price $\mathcal{K}$ then. As the gamma of a call option is typically given by a bell shaped curve centered near the call's strike, the greatest weight is given to the options whose strike is close to that of the target option. Furthermore, as we let the common maturity $u$ of the spanning portfolio approach the target call option's maturity $T$, the gamma becomes more concentrated around $K$. In the limit when $u=T$, all of the weight is on the call option of strike $K$, and equation (3) reduces to a tautology. 
Proof. Under the Markovian assumption in (1), the initial value of the target call option is given by discounting the expectation of the value it will have at the future date $u$ :

$$
\begin{aligned}
C(S, t ; K, T ; \Theta) & =e^{-r(u-t)} \int_{0}^{\infty} q(S, t ; \mathcal{K}, u ; \Theta) C(\mathcal{K}, u ; K, T ; \Theta) d \mathcal{K} \\
& =\int_{0}^{\infty} \frac{\partial^{2}}{\partial \mathcal{K}^{2}} C(S, t ; \mathcal{K}, u ; \Theta) C(\mathcal{K}, u ; K, T ; \Theta) d \mathcal{K} .
\end{aligned}
$$

The first line follows from the Markovian property: the call option value at any time $u$ depends only upon the underlying security's price at that time. The second line is obtained via a substitution of equation (2). We then integrate equation (5) by parts twice and observe the following boundary conditions,

$$
\frac{\partial}{\partial \mathcal{K}} C(S, t ; \infty, u ; \Theta)=0, \quad C(0, u ; K, T ; \Theta)=0, \quad C(S, t ; \infty, u ; \Theta)=0, \quad \frac{\partial}{\partial S} C(0, u ; K, T ; \Theta)=0 .
$$

The final result is as in equation (3).

Equation (3) represents a constraint imposed by no-arbitrage and the Markovian assumption on the relation between prices of options at different maturities. A violation of (3) implies an arbitrage opportunity. For example, suppose that at time $t$, the market price of a call option with strike $K$ and maturity $T$ (left hand side) exceeds the price of a gamma weighted portfolio of call options for some earlier maturity $u$ (right hand side). Then conditional on the validity of the Markovian assumption (1), the arbitrage is to sell the call option of strike $K$ and maturity $T$, and to buy the gamma weighted portfolio of all calls maturing at the earlier date $u$. The cash received from selling the $T$ maturity call exceeds the cash spent buying the portfolio of nearer dated calls. At time $u$, the portfolio of expiring calls pays off:

$$
\int_{0}^{\infty} \frac{\partial^{2}}{\partial \mathcal{K}^{2}} C(\mathcal{K}, u ; K, T ; \Theta)\left(S_{u}-\mathcal{K}\right)^{+} d \mathcal{K}
$$

Integrating by parts twice implies that this payoff reduces to $C\left(S_{u}, u ; K, T ; \Theta\right)$, which can be used to close the short call position.

To understand the implications of our theorem for risk management, suppose that at time $t$ there are no call options of maturity $T$ available in the listed market. However, it is known that such a call will 
be available in the listed market by the future date $u \in(t, T)$. An options trading desk could consider writing such a call option of strike $K$ and maturity $T$ to a customer in return for a (hopefully sizeable) premium. Given the validity of the Markov assumption, the risk exposure arising from writing the call option can be hedged away over the time period $[t, u]$ using a static position in available shorter term options. The maturity of the shorter term options should be equal to or longer than $u$ and the portfolio weight is determined by equation (3). Then at date $u$, the assumed validity of the Markov condition (1) implies that the desk can use the proceeds from the sale of the shorter term call options to purchase the $T$ maturity call in the listed market. Thus, this hedging strategy is semi-static in that it involves rolling over call options once. In contrast to a purely static strategy, there is a risk that the Markov condition (1) will not hold at the rebalancing date $u$. We will continue to use the terser term "static" to describe this semi-static strategy when it is contrasted to a classical dynamic strategy. However, we warn the practically minded reader that our use of this term does not imply that there is no model risk.

Theorem 1 states the spanning relation in terms of call options. One can readily show that the spanning relation also holds if one replaces the call options on both sides of the equation by their corresponding put options of the same strike and maturity. The relation on put options can either be proved analogously or via the application of put-call parity to the call option spanning relation in (3).

These static spanning relations stand in sharp contrast to traditional dynamic hedging strategies, which are based on continuous rebalancing of positions in the underlying asset. In what follows, we investigate the effectiveness of the two types of strategies using both Monte Carlo simulation and an empirical study.

\section{Finite Approximation with Gaussian Quadrature Rules}

In practice, one can neither rebalance a portfolio continuously, nor can one form a static portfolio involving a continuum of securities. Both strategies involve an infinite number of transactions and hence in the presence of discrete transaction costs, both would lead to financial ruin. Therefore, in reality, dynamic strategies are only rebalanced discretely. The trading times are chosen to balance the costs arising from the hedging error with the costs arising from transacting in the underlying. Similarly, 
to approximate our static hedging strategy, we need to use a finite number of calls. The number of calls is chosen to balance the cost from the hedging error with the cost from transacting in these options.

Specifically, we approximate the spanning integral in (3) by a weighted sum of a finite number $(N)$ of call options at strikes $\mathcal{K}_{j}, j=1,2, \cdots, N$,

$$
\int_{0}^{\infty} w(\mathcal{K}) C(S, t ; \mathcal{K}, u ; \Theta) d \mathcal{K} \approx \sum_{j=1}^{N} \mathcal{W}_{j} C\left(S, t ; \mathcal{K}_{j}, u ; \Theta\right)
$$

where we choose the strike points $\mathcal{K}_{j}$ and their corresponding weights based on the Gauss-Hermite quadrature rule.

The Gauss-Hermite quadrature rule is designed to approximate an integral of the form $\int_{-\infty}^{\infty} f(x) e^{-x^{2}} d x$, where $f(x)$ is an arbitrary smooth function. After some rescaling, the integral can be regarded as an expectation of $f(x)$ where $x$ is a normally distributed random variable with zero mean and variance of two. For a given target function $f(x)$, the Gauss-Hermite quadrature rule generates a set of weights $w_{i}$ and nodes $x_{i}, i=1,2, \cdots, N$ that are defined by

$$
\int_{-\infty}^{\infty} f(x) e^{-x^{2}} d x=\sum_{j=1}^{N} w_{j} f\left(x_{j}\right)+\frac{N ! \sqrt{\pi}}{2^{N}} \frac{f^{(2 N)}(\xi)}{(2 N) !}
$$

for some $\xi \in(-\infty, \infty)$. Note that the approximation error is reduced to zero if the integrand $f(x)$ can be represented as a $2 N-1$ degree polynomial function. See Davis and Rabinowitz (1984) for details.

To apply the quadrature rules, we need to map the quadrature nodes and weights $\left\{x_{i}, w_{j}\right\}_{j=1}^{N}$ to our choice of option strikes $\mathcal{K}_{j}$ and the portfolio weights $\mathcal{W}_{j}$. One reasonable choice of a mapping function between the strikes and the quadrature nodes is given by

$$
\mathcal{K}(x)=K e^{x \sigma \sqrt{2(T-u)}+\left(q-r-\sigma^{2} / 2\right)(T-u)},
$$


where $\sigma^{2}$ denotes the annualized variance of the $\log$ asset return. This choice is motivated by the gamma weighting function under the Black-Scholes model, which is given by

$$
\mathcal{W}(\mathcal{K})=\frac{\partial^{2} C(\mathcal{K}, u ; K, T ; \Theta)}{\partial \mathcal{K}^{2}}=e^{-q(T-u)} \frac{n\left(d_{1}\right)}{\mathcal{K} \sigma \sqrt{T-u}}
$$

where $n(\cdot)$ denotes the probability density of a standard normal and the standardized variable is defined as

$$
d_{1} \equiv \frac{\ln (\mathcal{K} / K)+\left(r-q+\sigma^{2} / 2\right)(T-u)}{\sigma \sqrt{T-u}}
$$

We thus can obtain the mapping in (8) by replacing $d_{1}$ with $x / \sqrt{2}$, which can also be regarded as a standard normal variable.

Thus, given the Gauss-Hermite quadrature $\left\{w_{j}, x_{j}\right\}_{j=1}^{N}$, we choose the strike points as

$$
\mathcal{K}_{j}=K e^{x_{j} \sigma \sqrt{2(T-u)}+\left(q-r-\sigma^{2} / 2\right)(T-u)},
$$

The portfolio weights are then given by

$$
\mathcal{W}_{j}=\frac{w\left(\mathcal{K}_{j}\right) \mathcal{K}_{j}^{\prime}\left(x_{j}\right)}{e^{-x_{j}^{2}}} w_{j}=\frac{w\left(\mathcal{K}_{j}\right) \mathcal{K}_{j} \sqrt{2} \sigma}{e^{-x_{j}^{2}}} w_{j} .
$$

The next section uses simulation to determine the effectiveness of this approximation.

\section{Simulation Analysis Based on Popular Models}

Consider the problem faced by the writer of a call option on a certain stock. For concreteness, suppose that the call matures in one year and is written at-the-money. The writer intends to hold this short position for a month, after which the option position will be closed. During this month, the writer can hedge his market risk using various exchange traded liquid assets such as the underlying stock, futures, and/or options on the same stock. In particular, we compare the performance of the following two strategies: (1) a static hedging strategy using one-month standard options, and (2) a dynamic 
delta hedging strategy using the underlying stock futures. The static strategy is based on the spanning relation in (3) and is approximated by a finite number of options, with the portfolio strikes and weights determined by the quadrature method. The dynamic strategy is discretized by rebalancing the futures position daily. The choice of using futures instead of the stock itself for the delta hedge is intended to be consistent our empirical study in the next section on S\&P 500 index options. For these options, direct trading in the stocks making up the index is infeasible, so all delta hedging is done in the very liquid index futures market. Given our assumption of constant interest rates and dividend yields, the simulated performances of the delta hedges based on the stock or its futures are almost identical. Hence, this choice does not affect our results.

We compare the performance of the above two strategies based on Monte Carlo simulation. For the simulation, we consider two data generating processes: the benchmark Black-Scholes model (BS) and the Merton (1976) jump-diffusion model (MJ). Under the objective measure, $\mathbb{P}$, the stock price dynamics in the two models are controlled by the following stochastic differential equations,

$$
\begin{array}{ll}
\text { BS } & d S_{t} / S_{t}=\mu d t+\sigma d W_{t}, \\
\text { MJ } & d S_{t} / S_{t}=(\mu-\lambda g) d t+\sigma d W_{t}+d J(\lambda),
\end{array}
$$

where $W$ denotes a standard Brownian motion in both models, and $J(\lambda)$ in the MJ model denotes a compound Poisson jump process with constant intensity $\lambda>0$. Conditional on a jump occurring, the MJ model assumes that the percentage jump is normally distributed with mean $\mu_{j}$ and variance $\sigma_{j}$, with the mean price change induced by a jump given by $g=e^{\mu_{j}+\frac{1}{2} \sigma_{j}^{2}}-1$.

While the data generating processes are specified under the objective measure $\mathbb{P}$, we also need to price the relevant options and compute the weights in the hedge portfolios, both of which are determined by the dynamics of the underlying security under a risk-neutral measure $\mathbb{Q}$. The risk-neutral dynamics of the above two models are assumed to take the following form,

$$
\begin{array}{ll}
\text { BS } & d S_{t} / S_{t}=(r-q) d t+\sigma d W_{t}^{*}, \\
\text { MJ } & d S_{t} / S_{t}=\left(r-q-\lambda^{*} g^{*}\right) d t+\sigma d W_{t}^{*}+d J^{*}\left(\lambda^{*}\right),
\end{array}
$$


where $W^{*}$ denotes a standard Brownian motion under measure $\mathbb{Q}$. The compound Poisson process under measure $\mathbb{Q}, J^{*}$, is assumed to have constant intensity $\lambda^{*}>0$. Conditional on a jump occurring, the jump size is normally distributed with mean $\mu_{j}^{*}$ and variance $\sigma_{j}^{2}$. See Bates (1991) for an equilibrium economy that supports such a measure change. For the simulation, we benchmark the parameter values of the two models to the S\&P 500 index. Specifically, we set $\mu=0.10, r=0.06$, and $q=0.02$ for both models. We further set $\sigma=0.27$ for the Black-Scholes model and $\lambda=\lambda^{*}=2.00, \mu_{j}=\mu_{j}^{*}=-0.10$, $\sigma_{j}=0.13$, and $\sigma=0.14$ for the Merton jump-diffusion model.

In each simulation, we generate a time series of daily underlying asset prices according to an Euler approximation of the respective data generating process. The starting value for the stock price is set to $\$ 100$. We simulate one months worth of data and consider a hedging horizon of one month. We assume that there are 21 business days in a month. To be consistent with the empirical study on S\&P 500 index options in the next section, we think of the simulation as starting on a Wednesday and ending on a Thursday four weeks later, spanning a total of 21 week days and 29 actual days. The hedging performance is recorded and, when needed, updated only on weekdays, but the interest earned on the money market account is computed based on actual over 360 . When simulating the sample paths, zero variation is assumed for the weekends.

At each week day, we compute the relevant option prices based on the realization of the security price and the specification of the risk-neutral dynamics. For the dynamic delta hedge, we also compute the delta each day based on the risk-neutral dynamics and rebalance the portfolio accordingly. For both strategies, we monitor the hedging error (profit and loss) at each week day based on the simulated security price and the option prices. The hedging error at each date $t, e_{t}$, is defined as the difference between the value of the hedging portfolio and the value of the target call option being hedged,

$$
\begin{aligned}
e_{t}^{D} & =B_{t-\Delta t} e^{r \Delta t}+\Delta_{t-\Delta t}\left(F_{t}-F_{t-\Delta t}\right)-C\left(S_{t}, t ; K, T\right) \\
e_{t}^{S} & =\mathcal{W}_{j} C\left(S_{t}, t ; \mathcal{K}_{j}, u\right)+B_{0} e^{r t}-C\left(S_{t}, t ; K, T\right),
\end{aligned}
$$

where the superscript $D$ and $S$ denote the dynamic and static strategies, respectively, $\Delta_{t}$ denotes the delta of the target call option with respect to the futures price at time $t, \Delta t$ denotes the amount of time 
between stock trades, and $B_{t}$ denotes the time- $t$ balance in the money market account. It includes the receipts from selling the one-year call option, less the cost of initiating and possibly changing the hedge portfolio. If one takes positions in call options of strikes as required by the static hedge, under no arbitrage, the value of the portfolio of the shorter term options should be equal to the value of the long term target option, and hence $B_{0}$ should be zero. However, since we use a finite number of call options in the static hedge to approximate the spanning relation, the bank account captures the value difference due to the approximation error, which is normally very small. Note that there is no rebalancing in the static strategy. We then compute the summary statistics of the hedge errors based on 1,000 simulations.

Under each model, the delta is given by the partial derivative $\partial C(S, t ; K, T ; \Theta) / \partial F$, with $F=$

$S e^{(r-q)(T-t)}$ denoting the forward/futures price. If an investor could trade continuously, this delta hedge removes all of the risk in the BS model. It does not remove all risk in the MJ model, but has nonetheless emerged as the market standard for implementing delta-hedges in jump models. The hedging portfolio for the static strategy is formed based on the weighting function $w(\mathcal{K})$ in $(4)$ implied by each model, the Gauss-Hermite quadrature nodes and weights $\left\{x_{i}, w_{i}\right\}$, and the mapping from the quadrature nodes and weights to the option strikes and weights, as given in equations (10) and (11). In computing the strike points, the annualized variance is $v=\sigma^{2}$ for the Black-Scholes model and $v=\sqrt{\sigma^{2}+\lambda\left(\mu_{j}^{2}+\sigma_{j}^{2}\right)}$ for the Merton jump-diffusion model. Given the chosen model parameters, $v \doteq 0.27^{2}$ for both models. Appendix A details the option pricing formula, the delta partial derivative $\partial C(S, t ; K, T ; \Theta) / \partial F$, and the weighting function $w(\mathcal{K})$ for both models.

\section{A. Hedging Comparison under the Diffusive Black-Scholes World}

The simulation results based on the Black-Scholes model are summarized in Panel A of Table I. Entries are the summary statistics of the hedging errors at the last step (at the end of the 21 business days) based on both strategies. For the dynamic strategy (the last column), we perform daily updating. For the static strategy, we consider hedge portfolios with $N=3,5,10,15,21$ one-month options. If the transaction costs in the options market and the underlying security market are comparable, we would expect that the transaction cost induced by buying 21 options at one time is close to the cost of buying or selling 
the underlying stock for 21 times. Hence, we would expect that performance of the daily updating delta hedge is comparable to the performance of the static hedge with 21 options.

For the static strategy, the simulation results indicate that the hedging performance improves as the number of options in the hedge increases. Nevertheless, the daily updating strategy beats the static strategy with 21 options in terms of the standard error, the root mean squared error (RMSE), the mean absolute error (MAE), and the mean short fall (MSF). The static strategy with 21 options does slightly better in terms of maximum profit or loss (Min and Max). Overall, the two strategies are comparable with a slight edge to the dynamic strategy. Thus, since the stock market is much more liquid than the stock options market, the dynamic delta strategy should be favored over the static strategy, if indeed stock prices move as in the Black-Scholes world,.

The hedge errors from the two strategies exhibit different distributional properties. In particular, the kurtosis of the hedging errors from the dynamic strategy is larger than that from all the static strategies. The kurtosis for the dynamic hedging errors is 4.68 , while that for the static hedges are below two in all cases. Therefore, when one is particularly concerned about avoiding large losses, the static strategy may be preferred.

The last row in each panel shows the accuracy of the Gauss-Hermite quadrature approximation of the integral in pricing the target options. Under the Black-Scholes model, the theoretical value of the target call is $\$ 12.35$, given under the dynamic hedging column. The approximation error is about one cent when applying a 21 node quadrature. The approximation error gradually increases as we reduce the number of quadrature nodes in the approximation.

\section{B. Hedging Comparison in Presence of Random Jumps as in the Merton World}

The hedging performance under the Merton jump-diffusion model is shown in Panel B of Table I. The performance of all the static strategies are comparable to their corresponding cases under the BlackScholes world. If anything, most of the performance measures for all the static strategies become slightly smaller under the Merton jump-diffusion case. In contrast, the performance of the dynamic 
strategy deteriorates dramatically as we move from the diffusion-based Black-Scholes model to the jump-diffusion process of Merton (1976). The standard error and the root mean squared error increase by a factor of ten for the dynamic strategy. The mean absolute error increases by a factor of four. As a result, the performance of the dynamic strategy is worse than the static strategy with merely three options.

Furthermore, the distributional differences between the hedge errors of the two strategies become more obvious under the Merton model. While the kurtosis of the static hedge errors remains small, the kurtosis of the dynamic hedge errors explodes from 4.68 in the BS model to 59.79 in the MJ model. The maximum loss from the dynamically hedged portfolio is $\$ 12.12$, even larger than the initial revenue from writing the call option (\$11.99). In contrast, the maximum loss is less than two dollars for the static hedge with merely three calls.

In the top two panels of Figure 1, we compare the simulated sample paths of the underlying security price under the two models. The daily movements under the Black-Scholes model are, usually small, while both small and large movements are quite evident under the Merton-jump diffusion model. The middle two panels of Figure 1 compare the sample paths of the hedging errors from the static hedging strategy using ten options. We apply the same scale for ease of comparison. While the sample paths of the static hedging errors look different under the two models, the relative magnitudes of the errors are similar. That is, the performance of the static hedging strategy is relatively insensitive to the specification of the underlying process. In contrast, the bottom two panels illustrate the sample paths of the dynamic hedging error under the two models. While the hedging errors are obviously smaller than the static ones under the Black-Scholes model (the scale remains the same), the hedging errors from the dynamic strategy explode under the Merton jump-diffusion model and we are forced to use a much larger scale in plotting the error paths. In particular, the large hedging errors from the dynamic strategy seem to correspond to the large moves in the underlying security price. Furthermore, most of the large errors are negative, irrespective of the direction of the large move in the underlying security price. This is because the option price function exhibits positive convexity with the underlying futures 
price. Under a large movement, the value of the delta portfolio is always below the value of the option contract. Thus, most of the large hedge errors for selling an option contract are losses (negative values).

Therefore, the daily delta hedging strategy performs reasonably well under the diffusion-based Black-Scholes model, but fails miserably when the underlying price jump randomly. In contrast, the performance of the static hedge with a few shorter term options is much less sensitive to the nature of the underlying price process. These simulation results parallel what theory predicts for continuously rebalanced delta hedges and for static hedges with a continuum of short term options. The continuously revised delta hedge was not designed to handle jumps of random size, while the static hedge with a continuum of short term options takes these jumps in stride. The discretizations needed to implement both strategies do not change the result that introducing jumps destroys the effectiveness of the delta strategy and has virtually no impact on our static hedging strategy.

\section{Effects of Model Uncertainty and Misspecification}

The above simulations are performed under the assumption that we know exactly under which model the options are priced. In practice, however, we can only use different models to approximately fit market option prices. Hence, model uncertainty is an inherent part of both pricing and hedging. To investigate the sensitivity of the hedging performance to model misspecification, we further compare the two types of hedging strategies when the hedger does not know the data generating process and hence must develop a hedging approach in the absence of this information. In particular, we assume that the actual underlying asset prices and the option prices are generated from the Merton jump diffusion model, but the hedge portfolios are formed using the Black-Scholes model, with an ad hoc adjustment using the observed implied volatility.

Specifically, for the static strategy, we compute the weighting function $w(\mathcal{K})$ based on the BlackScholes model, but use the eleven month at-the-money option implied volatility as the input for annualized volatility. For the dynamic strategy, the daily delta computation is based on the Black-Scholes formula using the implied volatility of the target call option as the volatility input. The practice of updating Black-Scholes deltas based on the market observed implied volatilities is in wide use in practice 

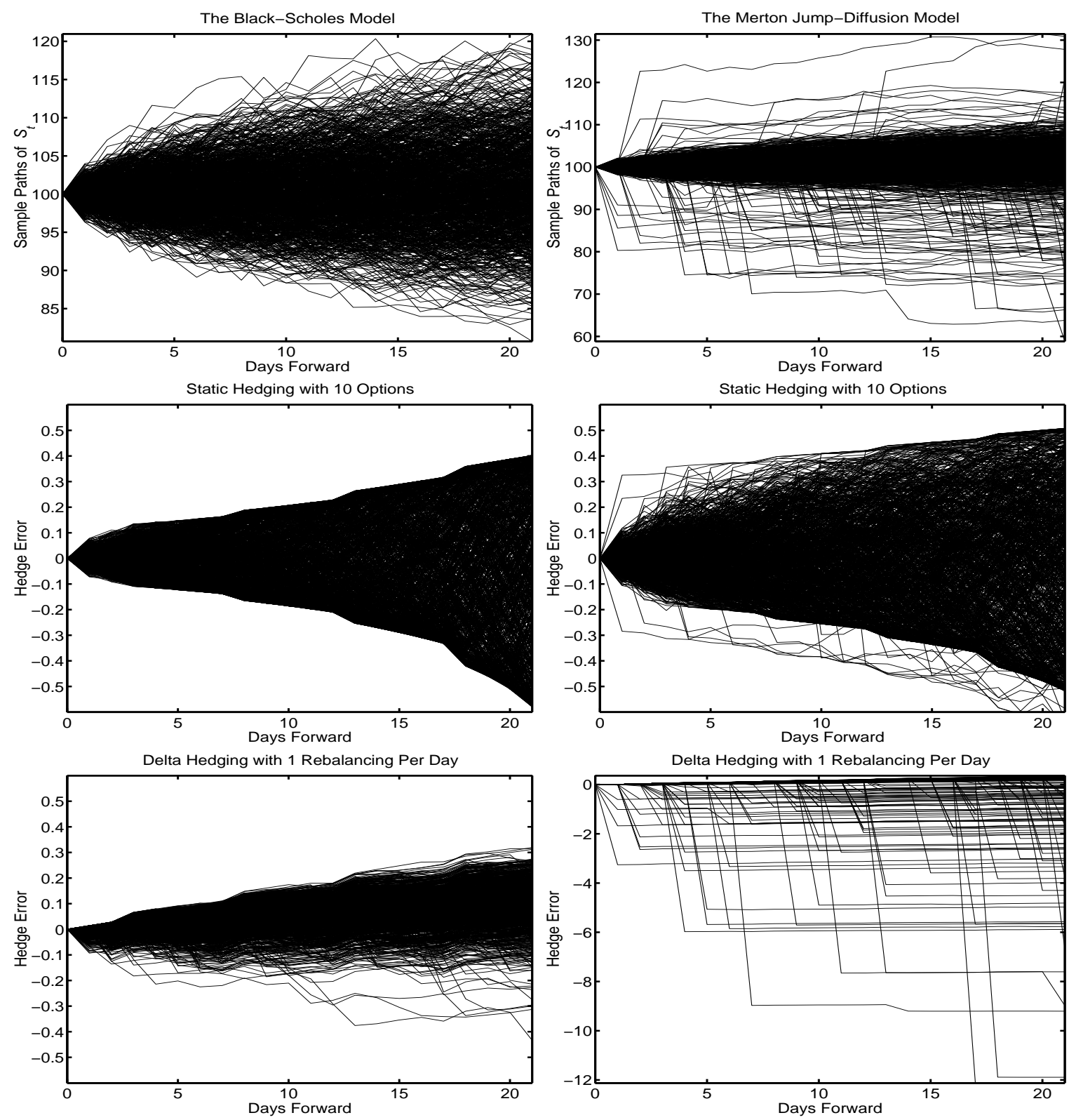

Figure 1. Hedging Performance Under Different Sample Paths

The two panels in the first row depicts the simulated sample paths for the underlying security price movement based on the Black-Scholes model (left) and the Merton jump-diffusion model (right). The second row depicts the sample paths of the hedging errors from the static hedging strategy with ten option contracts under the two models. The last row depicts the corresponding sample paths of the hedging errors from the dynamic delta strategy with the underlying futures and daily updating. 
as an ad hoc defense against model risk. Also, empirical studies in, for example, Engle and Rosenberg (1997), Jackwerth and Rubinstein (1996), and Bollen and Raisel (2002) have generally found that this approach works as well or better than the alternative approach of estimating a sophisticated model and delta-hedging with it. For the static hedge, we analogously assume that the hedger would use the Black Scholes gamma to pick strikes and strike weights with the initial implied volatility used as the volatility input.

The results are shown in Panel C of Table I. For the dynamic strategy, as long as we compute the delta based on the market implied volatility, the impact of model misspecification is minimal. For the static strategy, we observe some slight deterioration in performance when there are more than ten options contracts in the hedging portfolio, but the performance actually improves slightly when fewer options contracts are used in the hedge. Overall, model misspecification is not an over-riding concern in hedging.

These results are quite remarkable. They imply that in hedging, being able to span the right space is much more important than specifying the right model. For example, even if an investor has perfect knowledge of the stochastic process governing the underlying asset price, and hence can compute the perfectly correct delta, a dynamic strategy in the underlying asset still fails miserably in the presence of random jumps in the underlying asset price movement. In contrast, as long as an investor uses a few short term call options of different strikes in the hedge, the hedging error is about the same regardless of whether jumps can occur or not. This result holds even if the investor does not know which model to use to pick the appropriate strikes and portfolio weights.

\section{Effects of Rebalancing Frequency in Delta Hedging}

In the above simulations, we approximate the sample paths of the underlying stock price process using an Euler approximation with daily time steps and only consider dynamic delta strategies with daily updating. We are interested in knowing how much of the failure of the delta hedging strategy under the Merton jump-diffusion model is due to this somewhat arbitrary choice. Under the Black-Scholes environment, the dependence of the delta hedging error has been studied extensively in, for example, Black 
and Scholes (1972), Boyle and Emanuel (1980), Bhattacharya (1980), Derman and Kamal (1999), Galai (1983), Leland (1985), and Toft (1996). Several of these authors show that, under the Black-Scholes environment, the standard deviation of the hedging error arising from discrete rebalancing over a time step of length $\Delta t$ declines to zero slowly like $O(\sqrt{\Delta t})$. This subsection focuses on relating the hedging error to the rebalancing frequency under the Merton-jump diffusion model. Nevertheless, we also simulate the Black-Scholes model as a benchmark reference.

Table II illustrates the impacts of the rebalancing frequency on the hedging performance under three different cases: (A) the Black-Scholes model, (B) the Merton jump-diffusion model, both assuming that the hedger knows the underlying data generating process, and (C) ad hoc Black-Scholes delta hedging under the Merton world, where the hedger is assumed to not have knowledge of the data generating process. We consider cases where the rebalancing frequency increases from once per day, to twice per day, five times per days, and then to ten times per day. For ease of comparison, all the hedging exercises are performed on the same simulated sample paths. To accommodate the more frequent rebalancing, we now simulate the sample paths based on the Euler approximation with a time interval of 1/10-th of a business day. The slight differences between the dynamic hedging with daily updating in this table and in Table I is due to this difference in the simulation of the sample path.

Our simulation of the Black-Scholes model is consistent with the theoretical predictions in previous studies. As we increase the updating frequency from one to two, five, and ten times per day, the standard error of the hedging error reduces from 0.10 to $0.07,0.04$ and to 0.03 , adhering fairly closely to the $\sqrt{\Delta t}$ rule.

This speed of improvement in hedging performance is no longer valid, however, when the underlying data generating process is based on the Merton jump diffusion model, irrespective of whether the hedger knows the model or not. In the case when the process is known (Panel B), the standard error of the hedging errors remains around 1.02 and 1.03 as we increase the rebalancing frequency. In the ad hoc rebalancing case (Panel C), the standard error stays between 0.88 and 0.93 , against independent of the rebalancing frequency. Therefore, the failure of the delta hedging strategy under the Merton 
model is neither due to model misspecification, nor due to infrequent updating, but due to its inherent incapability in spanning risks associated with jumps of random size.

Note, however, that the Achilles heel of delta hedging in jump models is not the large size of the movement per se, but rather it is the randomness of the jump size at the jump time. For example, Cox and Ross (1976) and Dritschel and Protter (1999) show that dynamic delta hedging can span all risks arising in their pure jump models $\underset{i}{ }$ However, just before any jump can occur, its possible size is known at that time. This is analogous to the binomial model where again only two subsequent asset prices are possible. Under both cases, delta hedging can remove all risk. Therefore, it is the property that the jump size is unknown ex ante that is the source of the diffiuclty in dynamic delta hedging.

\section{E. Effects of Target and Hedging Instrument Choice}

For concreteness, the above simulations focused on the hedging of a one-year call option. Furthermore, although the static spanning relation permits many option maturities in the spanning portfolio, we only used one-month options. In this subsection, we compare the hedging performance when we choose different target options being hedged and different maturities for the options in the static hedging portfolio. In theory, if we use a continuum of options at a certain maturity, the spanning is perfect regardless of the exact maturity choice for the hedging portfolio. In practice, however, the gaussian quadrature approximation error may be different in different cases. The simulation analyzes how the hedging error introduced by the quadrature approximation varies over different choices of target and hedging options. Along the same line, we also analyze how the dynamic hedging error varies with the choice of the target option.

The results are reported in Table III. To save space, we only report static hedges with three and five options and compare their performance with that of delta hedging with daily updating. First, we investigate the impact of the target option maturity given the same hedging instruments. We choose target option maturities of two months, four months, and 12 months. For the static hedging strategy, as we lower the target option's maturity, the hedging errors get smaller in the Black-Scholes model 
but larger in the Merton jump diffusion model. We conjecture that these variations in performance are related to the different accuracies of the quadrature approximation for different integrands.

For the dynamic strategy, the hedging errors are larger for hedging shorter term options than for hedging longer term options under all simulated scenarios. This deteriorating performance with declining maturity is probably linked to the gamma of the target option. The shorter the maturity, the larger is the gamma is for an at-the-money option. Since the delta strategy can be regarded as a linear approximation, the hedging error normally increases with gamma, especially in the presence of large moves.

Our static spanning relation permits different maturities in forming the static hedges. Thus, holding the same one-year option as the target option, we also compare how different maturity options fare in spanning the risk of this target option. Under all three scenarios, we find that the hedging performance improves quite significantly when one increases the maturity of the hedging options. For example, under the Black-Scholes environment, the standard error of the hedging error is 0.66 when we use five one-month options to hedge the one-year option. This performance is much worse than daily delta hedging, which generates a standard error of merely 0.10 . However, as the one-month options in the portfolio are replaced by two month options and then by four month options, the performance of the static hedge improves quite dramatically. The standard error of the hedging error declines to 0.25 when using two month options and to a meager 0.04 when using four month options. Thus, when moderately longer term options are liquid and available in the market, we can further improve the performance of the static hedging strategy such that it outperforms daily delta hedging even under the Black-Scholes environment. Comparing this to Table II, the static hedging error of 0.04 is equivalent to a dynamic delta strategy of updating five to ten times per day!

The same trend follows under the Merton jump diffusion world, although the improvement in performance is not as dramatic. For example, under the Merton world, the standard error of the hedging error is 0.47 when hedging one-year options with five one-month options. The error is reduced to 0.29 when using five two-month options and is further reduced to 0.16 when using five four-month options, which is much smaller than the standard error of the dynamic hedging error (1.05) under daily updating. 
In short, the simulation exercises illustrate that particularly when the underlying asset price movement exhibits random jumps, our static strategy with a few appropriately chosen options can deliver much smaller hedging errors than does the dynamic delta strategy. But probably the biggest advantage of the static strategy lies in its flexibility. For the same target option, we have the freedom to choose options at different maturities to form the hedging portfolio. Furthermore, while the Gauss-Hermite quadrature rule provides a convenient way in performing finite approximations, there is ample room left for future research in developing better approximating schemes that can further improve the performance of the static strategy. Possible research directions include having multiple maturities in the static hedge and using delta hedging to further reduce the risk remaining from static hedging.

The fact that a static hedging strategy with merely three to five options can outperform a dynamic strategy with daily updating is remarkable. In addition to the above mentioned flexibility and potentially reduced transaction costs due to fewer transactions, there are several other advantages in implementing the static strategy. First, since the static hedge employs neither short stock positions nor substantial borrowing, ${ }^{1}$ it is not subject to either short sales restrictions or leverage constraints. In contrast, delta hedges of options always involve a short position in either the risky asset or a riskfree bond, and hence always face one of these restrictions. Second, while we do not explicitly consider transactions costs in the determination or implementation of either kind of hedge, we note that for certain underlying assets such as electricity or weather, one cannot even trade the underlying directly. Finally, the use of a static hedge also allows one to economize on the monitoring costs (e.g., paying for traders and real time data feeds) associated with dynamic rebalancing.

\section{Hedging S\&P 500 Index Options: An Applied Example}

The simulation study in the previous section compares the performance of the two different types of hedging strategies under controlled conditions. In this section, we investigate the historical perfor-

\footnotetext{
${ }^{1}$ The money market account induced by the approximation error for the static strategy is normally very small, and can be reduced to zero via a rescaling of portfolio weights without much effect on the hedging performance.
} 
mance of the two hedging strategies in hedging S\&P 500 index options. While the simulations allow us to benchmark the magnitude of the approximation error in various Markov models, only an empirical study can gauge the likely effectiveness of the two types of hedging strategies when applied in practice. Furthermore, since the simulations indicate that the two strategies exhibit comparable performance under the Black-Scholes world but the static strategy is much better for handling price jumps, the relative performance of the two strategies in the past can also serve as an indirect test on whether the S\&P 500 index moves diffusively or with jumps.

\section{A. Data and Estimation}

The data on S\&P 500 index options are obtained from OptionMetrics, a financial research and consulting firm specializing in econometric analysis of the options markets. The "Ivy DB" data set from OptionMetrics is the first widely-available, up-to-date, and comprehensive source of high-quality historical price and implied volatility data for the US equity and index options markets. Encompassing six years of data, Ivy DB contains accurate historical prices of options and their associated underlying instruments, correctly calculated implied volatilities, and option sensitivities. The index options data we have obtained from OptionMetrics are from January 1996 to August 2002. The data sets includes, among other information, the closing quotes on each options contract (bid and ask) and implied volatilities based on the mid quote. Also included in the data set is a unique option contract identifier to facilitate the tracking of an option contract over time. The underlying index level at close, the interest rate curve, and projected dividend yield for the calculation of implied volatility are also supplied by OptionMetrics. Our hedging exercises are based on the mid option price quotes.

To mimic the hedging strategies in the simulations, we perform month-long hedging exercises on the index options. The S\&P 500 index options expire on the Saturday following the third Friday. Since the terminal payoff is computed based on the opening price on that Friday morning, trades and quotes on the expiring options effectively stop on the preceding Thursday. Hence, we start the hedging exercise each month 30 days prior to the expiring Friday, which is a Wednesday. The available number of one-

month option contracts at each of the starting dates ranges from 48 to 142, half of them call options and 
half of them put options. From these starting dates, we can perform monthly hedging exercises for 79 non-overlapping months, from January 1996 to July 2002. Sampling properties of the hedging errors can then be computed from the 79 hedging experiments.

At each starting date, we consider options at four maturity groups, which try to match those used in the simulation: (1) one-month options, (2) two-month options, (3) options with maturities four to six months, and (4) options with maturities twelve to seventeen months. The variation in maturities in the last two maturity groups was needed to obtain a monthly series. Just as in the simulation exercise, we use the last three groups (two, four, and twelve month options) for target options being hedged and the first three groups (one, two, and four month options) in forming static hedging portfolios. The target option strikes are chosen to be close to the spot index level at the starting date.

Since we do not know what the "true" data generating process or option pricing model, we adopt an ad hoc strategy using the Black-Scholes model. For the dynamic strategy, we delta hedge with the underlying futures based on the Black model, using the observed implied volatility to compute the delta. For the static strategy, the portfolio is formed based on the Black-Scholes formula, with the at-the-money implied volatility of the appropriate maturity as the annualized variance input. When quotes at the appropriate strikes are not available, we use the nearest available strike contract as a replacement. For the static strategy, we can pick any number of shorter term options based on the quadrature rule. However, a large order quadrature rule often requires some deep out-of-the-money or deep in-the-money option contracts that are not available on the market. Thus, we focus on analyzing the performance of the static hedge with only three to five option contracts.

We follow both strategies from the starting date to the Thursday of the third following week, the last day of trading for the one-month options used in the static hedge, for 29 actual days. For the static strategy, we only need to track the price of the short term options at each date and record the difference between the price of the hedge portfolio and the price of the target call option. When there is a discrepancy between the price of the target option and the cost of the quadrature-determined hedge portfolio, we also monitor the typically small money market account balance. For the dynamic strategy, we need to compute the new delta at each date based on the newly observed underlying price level 
and implied volatility and perform the appropriate rebalancing. For ease of comparison, we align the hedging errors based on the weekdays of each week and then compute the sample properties of the hedging errors at each week day.

\section{B. Hedging Performance in Practice}

Figure 2 depicts the normalized sample paths of the S\&P 500 index level over the 79 month-long hedging experiments. The four major breaks in the sample paths reflect the four weekends of the month. There may also be other breaks due to holidays. When we compare this to the simulated sample paths under the Black-Scholes model and the Merton model, we see that the index's sample paths exhibit both small and large movements. The jumps, however, are not as dramatic as those shown on the simulated paths of the Merton jump-diffusion model.

Figure 3 depicts the 79 sample paths of the hedging errors for the hedging of at-the-money options at maturities of (1) two months (top row), (2) four-six months (middle row) and (3) one year or longer (bottom row). The horizontal axis is based on the actual number of days forward. Again, the four breaks in the sample paths represent the four weekends during the month long monitoring of the hedging performance. One also observes occasional path breaks during the weekdays, which can be either due to holidays or missing data: The delta is updated only when the market quote for the target option being hedged is available on that day. The performance of the static hedging is recorded only when the market quotes for all the relevant options (the options in the hedge portfolio and the target call option) are available.

The three panels on the left are hedging errors based on the static hedging strategy with a portfolio of three one-month options, in the middle are based on static hedging with a portfolio of five one-month options, and on the right are errors based on the dynamic delta hedging strategy with the underlying futures and daily updating. For ease of comparison, we apply the same scale on all panels in the figure. Overall, the performance of the static strategy with a portfolio of merely three to five options is comparable to the performance of the daily updating delta hedging strategy. Furthermore, the hedging performance of the dynamic delta strategy is relatively stable as we hedge options of different maturi- 


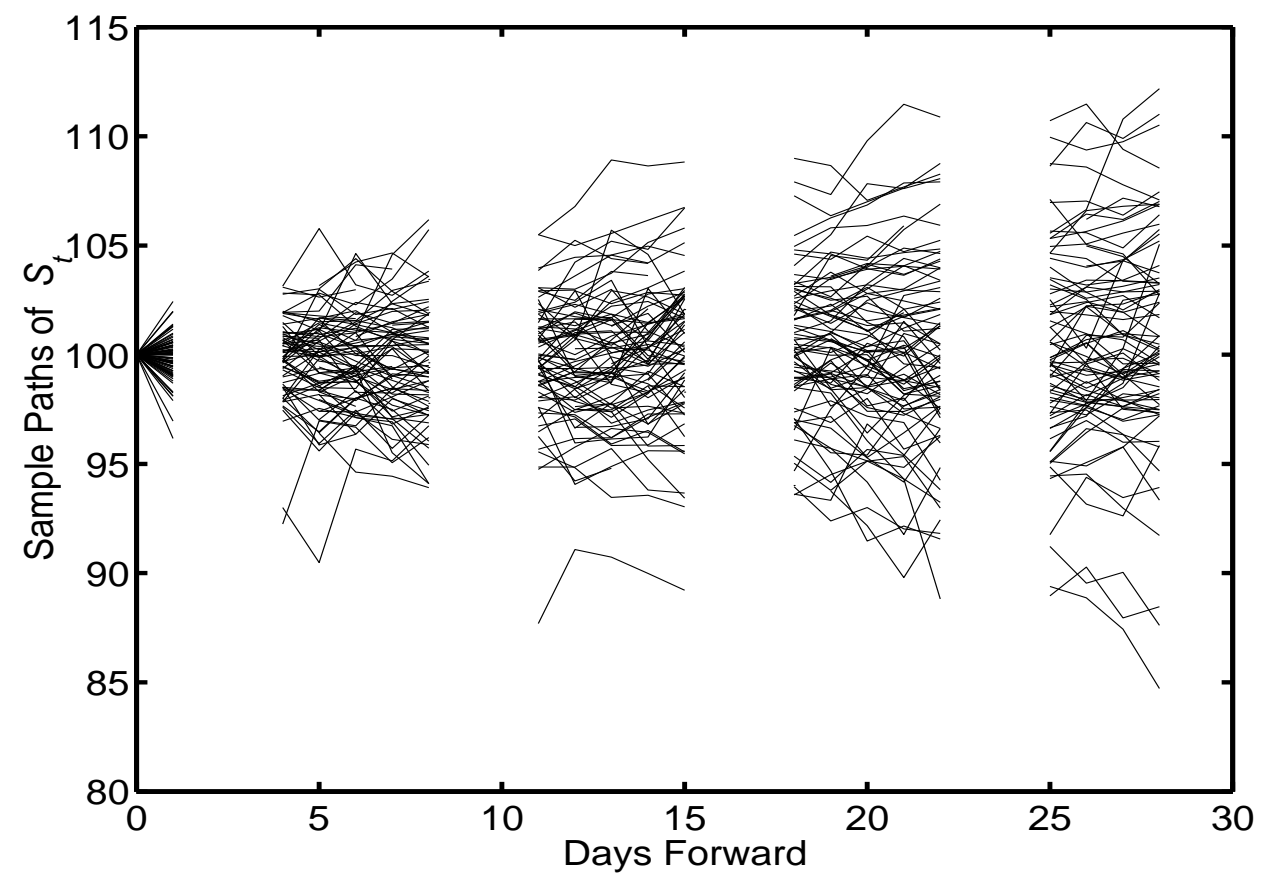

Figure 2. Normalized Sample Paths of the S\&P 500 Index

Plots are the sample paths of the S\&P 500 index level over the month-long horizon of the hedging exercises. The index level is normalized to 100 at the start of each hedging exercise. The data are aligned based on weekdays, starting on a Wednesday and ending on a Thursday five weeks later, altogether 29 days. 

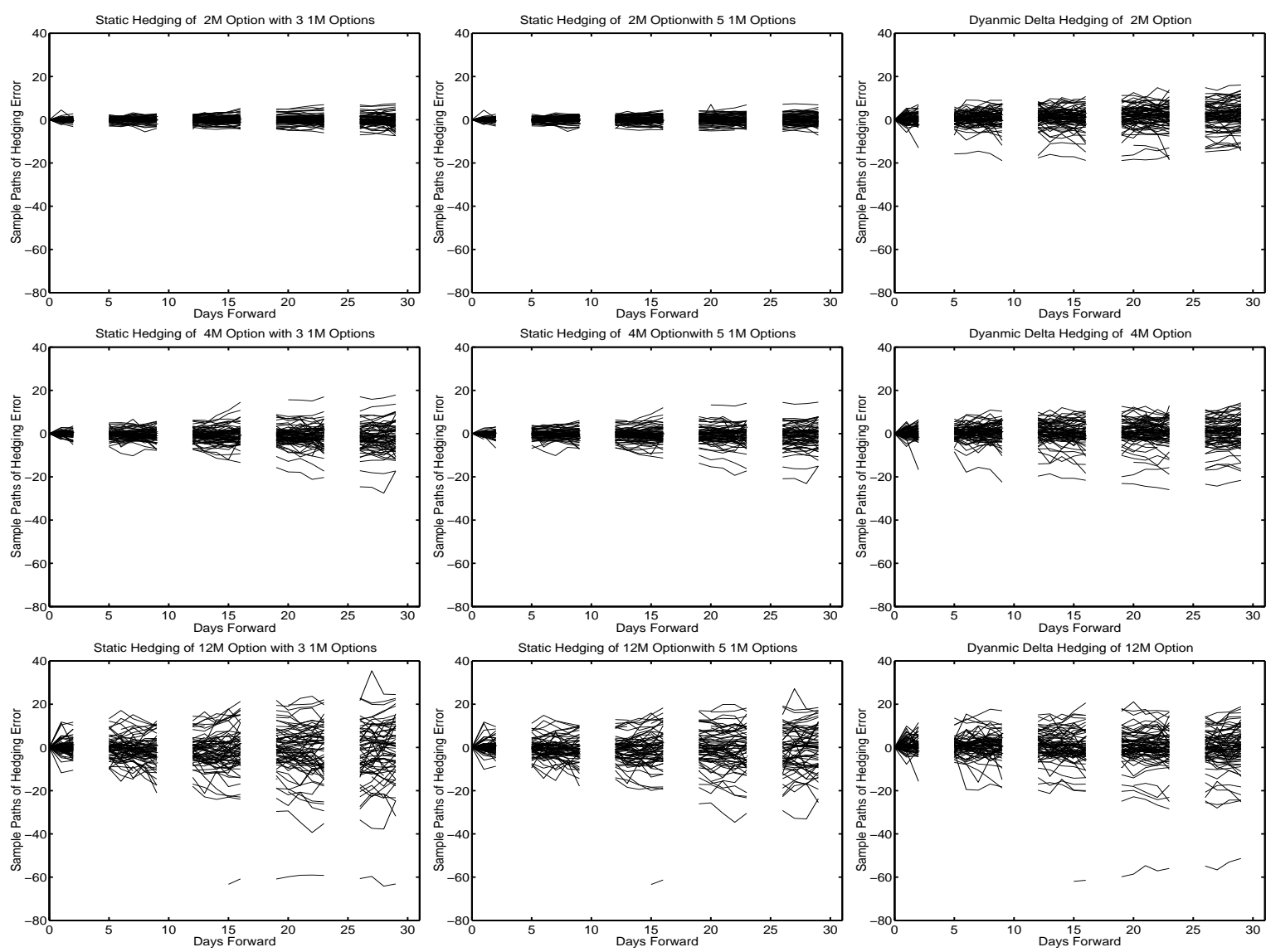

Figure 3. Hedging Errors from Static and Dynamic Strategies

Plots are the sample paths of the hedging errors based on (1) static strategies with a portfolio of three one-month options (left column), (2) static strategies with a portfolio of five one-month options (middle column) and (3) dynamic delta hedging strategies with daily updating with the underlying futures (right column). The options being hedged are at the money and have maturities of (1) two months (top row), (2) four-six months (middle row), and (3) one year and longer (bottom row). 

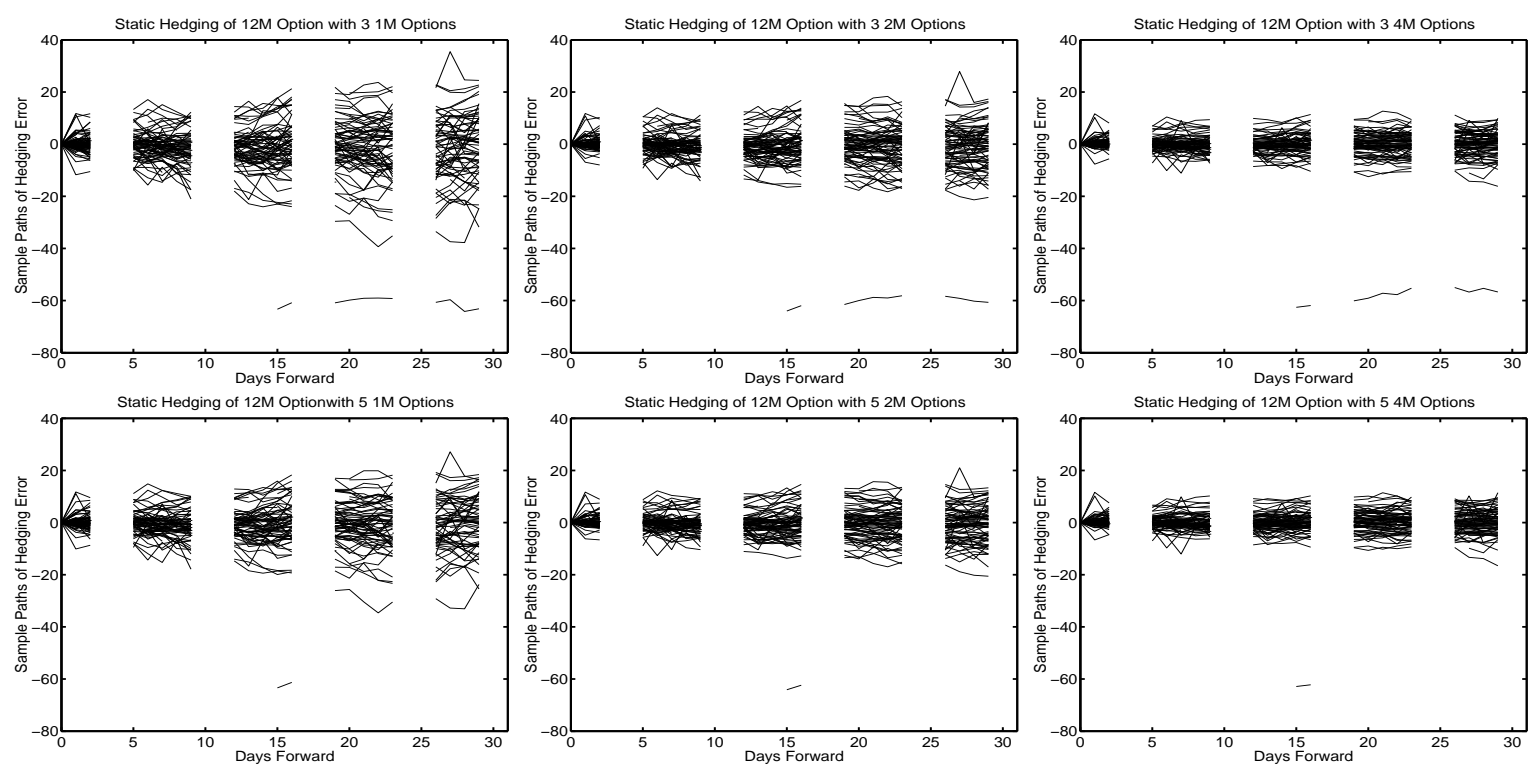

Figure 4. Errors of Static Hedging With Options of Different Maturities

Plots are the sample paths of the hedging errors for the static hedging strategy with a portfolio of three options. The maturity of the options being hedged is one year or longer. The maturities of options in the hedging portfolio are (1) one month (left), (2) two months (middle), and (3) four-six months (right). The hedging portfolio contains three options in the first row, and five options in the second row.

ties, but the one-month options seem to hedge the two-month options much better than they hedge the four-month and one-year options. As such, the performance of the one-month option static hedging portfolio performs better than the dynamic delta strategy in hedging two-month options, but slightly worse off in hedging the one-year options.

In Figure 4, we compare the performance of the static hedging strategy when the target call option is fixed at one-year or longer maturity, but the hedging portfolio is made of options at different maturities. From left to right, the maturities from the options in the hedging portfolio increases from one month to two month (middle) and then to four to six months (right panel). The top panels are based on portfolios with three options while the bottom panels are based on portfolios with five options. We observe that from left to right, as the maturity of the options in the hedging portfolio increases, the hedging error declines. This result is consistent with the simulation result in showing that relatively longer term options are more effective in spanning the target option when a quadrature approximation is applied in setting up the portfolio. 
Table IV summarizes the mean absolute hedging errors of these hedging exercises at each weekday of the month-long hedging horizon. When using a portfolio of three one-month options to perform the static hedge, the mean absolute hedging errors at the closing date (the last Thursday) is 2.42 when hedging the two-month options, 5.73 when hedging the four-six month options, and 10.85 when hedging options one year or longer. The corresponding mean absolute hedging errors are smaller when using five options. They are, respectively, 2.08, 4.35, and 8.20. On the other hand, given the same one-year or longer option to be hedged, the mean absolute hedging errors decline as we use longer maturity options to form the hedging portfolio. When using three options in the hedging portfolio, the mean absolute hedging error declines from 10.85 to 7.42 , and then to 4.61 as the maturity of the options in the hedging portfolio increases from one month to two months and then to four to six months. When using five options in the hedging portfolio, the decline is from 8.20 to 5.74 and then to 3.74 . In contrast, the mean absolute hedging errors of the daily delta strategy with the underlying futures is between 5.38, 5.38, and 7.35 for hedging two month, four to six month, and one year or longer options. Thus, overall an appropriately chosen static hedging strategy with merely three options can outperform the dynamic delta hedging strategy with the underlying futures.

\section{Empirical Implications on the Index Movement}

Comparing the empirical results in Table IV with the simulation result in Table III, we obtain a few interesting implications. First, the fact that a static strategy with merely three options can outperform the daily delta hedging implies that the data generating process does not follow the geometric Brownian motion as specified in the Black-Scholes model. The comparison lends empirical support to the likely existence of random jumps in the S\&P 500 index movement. This result is consistent with the findings from many parametric studies, and also with the results from the more generic tests such as Ait-Sahalia (2002) and Carr and Wu (2001).

Second, in agreement with the simulation, the static hedging performance improves as the maturity of the options in the hedging portfolio increases. But the results from the data contrast with the simulation results as we vary the maturity of the target options. Overall, the simulations indicate that the 
performance from the dynamic strategy is better in hedging long term options than in hedging short term ones, potentially due to the difference in the option's gamma. In contrast, the results from our data analysis imply that the hedging performance of the dynamic strategy is quite stable as we change the target call option's maturitu. In particular, the mean absolute hedging error is actually higher when hedging one year options than when hedging shorter term options. The same discrepancy applies to the static strategy. Under simulated scenarios with jumps, the static hedging strategy also performs better in hedging long term options than hedging shorter ones, again in contrast to the observation on S\&P 500 options. Indeed, the historical performance actually deteriorates quite significantly from statically hedging two month options to hedging one year options. This last observation prompts us to conjecture that there are additional sources of risk that are also affecting the option prices. One such risk could be stochastic volatility. When there are additional sources of risk, the hedging performance may deteriorate as the hedging portfolio and the target call exhibit different exposures to the additional risk sources. For example, when the static hedging portfolio matures, the resulting payoff is purely determined by the realized index level and does not depend upon any other state variables such as volatility. Nevertheless, the value of the unexpired target call option may be sensitive to the volatility level at that time. This different sensitivity to other sources of risk could create a deterioration in the hedging performance. The improved performance of the static hedging strategy when the hedging option maturity increases also supports such a conjecture.

\section{When Does a Hedge Fail?}

While the mean absolute hedging error provides us with a general measure on how each strategy performs in hedging the index options, it is interesting to know under what scenarios a hedging strategy is most likely to fail. For this performance, at each weekday of the month-long hedging exercise sample, we identify the month at which the absolute hedging error is largest. The results are summarized in Table V. The numbers in the table represent the months under which the absolute hedging error is the largest at that specific weekday. The corresponding starting date of each identified month is listed in

Table VI. For the dynamic delta hedge, the most prominent failing month is the 22nd month of our 
sample, which starts on October 23, 1997. During this month, the impact of the Asian crises was finally felt in the United States stock market. The Dow Jones Industrial Average dropped by more than 500 points in a Monday and the stock market was forced to halt trading twice in a day. During the next day, the market bounded back dramatically, followed by large seesaw movements in the market. The dynamic delta strategy was obviously having big troubles managing the risk induced by such large movements and hence generated a series of hedging errors that are the largest in the whole sample. In contrast, this month is rarely identified in the static hedging case as the worst performing month. This means that the static strategy deals well with these large market movements during crisis times. The worst performing months for the static hedges are not associated with the known financial crises mentioned in the introduction.

\section{Concluding Remarks}

To hedge the risk associated with the sale of a given option, we develop a static hedging strategy using a portfolio of nearer dated options. The portfolio is designed to hedge the risk associated with jumps of random size, a source of risk that cannot be dealt with by delta hedging. Since a perfect static hedge requires a continuum of strikes, we develop a discrete approximation of the static hedge and tests its effectiveness using simulations. The simulations indicate that the static hedge approximation has about the same effectiveness as delta hedging in the Black-Scholes environment with daily rebalancing. However, as soon the simulated underlying price process can also experience jumps of random size, the performance of the delta hedge deteriorates dramatically, even though the delta is chosen with full knowledge of the stochastic process. In contrast, the performance of our static strategy with options is relatively insensitive to the change from a purely diffusive process to a jump diffusion. These conclusions were unchanged when we switched to ad hoc static and dynamic hedges necessitated by a lack of knowledge of the driving jump diffusion process. Further simulation indicates that the inferior performance of the delta hedge in the presence of jumps cannot be improved by increasing the rebalancing frequency, but the superior performance of the static hedging strategy can be further enhanced with the flexible choice of option maturities in the hedging portfolio. As a result, superior risk reduction was 
achieved by the static hedge in this setting with as few as three options in the portfolio. Thus, also accompanying the superior performance are the potentially lower transaction and monitoring costs. Furthermore, since delta hedging also requires short positions in either the risky asset or the riskfree one, the complications arising from short sales restrictions and leverage constraints are completely circumvented.

To investigate how our ad hoc static strategy performs in a realistic setting, we investigate its effectiveness in hedging S\&P 500 index options and compare its performance with ad hoc daily delta hedging in the index futures. We find that the superior performance of our ad hoc static hedge found in the simulations of the Merton model also extends to the index options data. This finding lends indirect support to the existence of jumps of random size as part of the S\&P 500 index dynamics. We also find that the performance of our ad hoc static hedge is best when the maturity of the options in the hedging portfolio is close to the maturity of the option being hedged. As the maturity gap increases, the hedging performance deteriorates moderately, indicating the potential existence of additional factors affecting option prices. One such risk can come from stochastic volatility. Hence, based on the availability and liquidity of the relevant option contracts, future research should be directed towards developing and testing static hedges which account for a second risk factor. Such strategies would in general involve simultaneous positions in multiple strikes and maturities.

One can also investigate effective ways to deal with the complications introduced by illiquidity in options markets. If an insufficient number of strikes are available at each maturity nothing prevents combining options of multiple maturities. Another avenue for increasing liquidity is to consider options on other highly correlated underlying assets. For example, static positions in OEX options might by used to supplement static positions in S\&P 500 options, provided that the methodology can be extended to deal with the complications arising from optimal early exercise. Finally, to reduce the risk remaining after an optimal static hedge has been found, dynamic strategies can be employed in conjunction. In the interests of brevity, the development and testing of such strategies is deferred to future research. 


\section{Appendix A. Option Pricing and Hedging under BS and MJ models}

\section{Appendix A.1. The Black-Scholes Model}

Under the Black-Scholes model, the spot price follows geometric Brownian motion, under measure $\mathbb{Q}$,

$$
d S_{t} / S_{t}=(r-q) d t+\sigma d W_{t}^{*}
$$

The time- $t$ value of a European call with strike $K$ and maturity $T$ is given by

$$
C(S, t ; K, T ; \Theta)=S e^{-q(T-t)} N\left(d_{1}(S, t ; K, T ; r, q, \sigma)\right)-K e^{-r(T-t)} N\left(d_{1}(S, t ; K, T ; r, q, \sigma)-\sigma \sqrt{T-t}\right)
$$

where $N(\cdot)$ denotes the standard normal distribution function and

$$
d_{1}(S, t ; K, T ; r, q, \sigma)=\frac{\ln (S / K)+\left(r-q+\sigma^{2} / 2\right)(T-t)}{\sigma \sqrt{T-t}} .
$$

The delta and weighting function are given by

$$
\begin{aligned}
\Delta & \equiv \frac{\partial C(S, t ; K, T ; \Theta)}{\partial F}=e^{-r(T-t)} N\left(d_{1}(S, t ; K, T ; r, q, \sigma)\right), \\
w(\mathcal{K}) & =\frac{\partial^{2} C(\mathcal{K}, u ; K, T ; \Theta)}{\partial \mathcal{K}^{2}}=e^{-q(T-u)} \frac{n\left(d_{1}(\mathcal{K}, u ; K, T ; r, q, \sigma)\right)}{\mathcal{K} \sigma \sqrt{T-u}},
\end{aligned}
$$

where $n(\cdot)$ denotes the probability density function of a standard normal,

$$
n\left(d_{1}\right)=\frac{1}{\sqrt{2 \pi}} e^{-d_{1}^{2} / 2}
$$

Given the Gauss-Hermite quadrature $\left\{x_{j}, w_{j}\right\}_{j=1}^{N}$, the static portfolio strikes and weights are given by,

$$
\begin{aligned}
\mathcal{K}_{j} & =K e^{x_{j} \sigma \sqrt{2(T-u)}+\left(q-r-\sigma^{2} / 2\right)(T-u)}, \\
\mathcal{W}_{j} & =\frac{w\left(\mathcal{K}_{j}\right) \mathcal{K}_{j} \sigma \sqrt{2(T-u)}}{e^{-x_{j}^{2}}} w_{j}=\frac{e^{-q(T-u)}}{\sqrt{\pi}} w_{j} .
\end{aligned}
$$

Note that given the definition of $\mathcal{K}_{j}$, we have $d_{1}\left(\mathcal{K}_{j}, u ; K, T\right)=\sqrt{2} x_{j}$ and hence

$$
n\left(d_{1}\left(\mathcal{K}_{j}, u ; K, T ; r, q, \sigma\right)\right)=\frac{1}{\sqrt{2 \pi}} e^{-x_{j}^{2}} .
$$


The cancellation then follows for the portfolio weight $\mathcal{W}_{j}$.

\section{Appendix A.2. The Merton Jump-Diffusion Model}

Merton (1976)'s Poisson jump-diffusion model assumes the following risk-neutral dynamics for the underlying security price movement,

$$
\left.d S_{t} / S_{t}=r-q-\lambda^{*} g^{*}\right) d t+\sigma d W_{t}^{*}+d J^{*}\left(\lambda^{*}\right)
$$

where $d J^{*}$ denotes a compound Poisson jump with intensity $\lambda^{*}$. Conditional on one jump occurring, the percentage jump size is normally distributed with mean $\mu_{j}^{*}$ and variance $\sigma_{j}^{2}$. The mean price change per one jump is given by $g^{*}=\left(e^{\mu_{j}^{*}+\sigma_{j}^{2} / 2}-1\right)$.

The price of a European call can be written as a weighted average of the Black-Scholes option price function, with the weight given by the Poisson probability of jumps,

$$
C(S, t ; K, T ; \Theta)=e^{-r(T-t)} \sum_{n=0}^{\infty} \operatorname{Pr}(n)\left[S e^{\left(r_{n}-q\right)(T-t)} N\left(d_{1 n}(S, t ; K, T)\right)-K N\left(d_{1 n}(S, t ; K, T)-\sigma_{n} \sqrt{T-t}\right)\right],
$$

where $\operatorname{Pr}(n)$ is the probability of having $n$ jumps between time $t$ and $T$,

$$
\operatorname{Pr}(n)=e^{-\lambda^{*}(T-t)} \frac{\left(\lambda^{*}(T-t)\right)^{n}}{n !}
$$

$d_{1 n}(S, t ; K, T)$ is defined as

$$
d_{1 n}(S, t ; K, T)=\frac{\ln (S / K)+\left(r_{n}-q-\sigma_{n}^{2} / 2\right)(T-t)}{\sigma_{n} \sqrt{T-t}}
$$

with

$$
\begin{aligned}
r_{n} & =r-\lambda^{*} g^{*}+n\left(\mu_{j}^{*}+\sigma_{j}^{2} / 2\right) /(T-t), \\
\sigma_{n}^{2} & =\sigma^{2}+n \sigma_{j}^{2} /(T-t) .
\end{aligned}
$$

The delta and weighting function are given by

$$
\Delta=e^{-2 r(T-t)} \sum_{n=0}^{\infty} \operatorname{Pr}(n) e^{r_{n}(T-t)} N\left(d_{1 n}(S, t ; K, T)\right),
$$




$$
w(\mathcal{K})=e^{-r(T-u)} \sum_{n=0}^{\infty} \operatorname{Pr}(n) e^{\left(r_{n}-q\right)(T-u)} \frac{n\left(d_{1 n}(\mathcal{K}, u ; K, T)\right)}{\mathcal{K} \sigma_{n} \sqrt{T-u}}
$$

We define the strike price points based on the Gauss-Hermite quadrature $\left\{x_{j}, w_{j}\right\}_{j=1}^{N}$ as follows,

$$
\mathcal{K}_{j}=K e^{x_{j} \sqrt{2 v(T-u)}+(q-r-v / 2)(T-u)},
$$

where

$$
v=\sigma^{2}+\lambda^{*}\left(\left(\mu_{j}^{*}\right)^{2}+\sigma_{j}^{2}\right)
$$

is the annualized variance of the asset return under measure $Q$. The portfolio weights are then given by

$$
\begin{aligned}
\mathcal{W}_{j} & =\frac{w\left(\mathcal{K}_{j}\right) \mathcal{K}_{j} \sqrt{2 v(T-u)}}{e^{-x_{j}^{2}}} w_{j}, \\
& =\frac{\sqrt{2 v}}{e^{-x_{j}^{2}}} w_{j} e^{-r(T-u)} \sum_{n=0}^{\infty} \operatorname{Pr}(n) e^{\left(r_{n}-q\right)(T-u)} \frac{n\left(d_{1 n}\left(\mathcal{K}_{j}, u ; K, T\right)\right)}{\sigma_{n}} \\
& =\left[\frac{1}{\sqrt{\pi}} \sum_{n=0}^{\infty} \operatorname{Pr}(n) e^{\left(r_{n}-r-q\right)(T-u)} e^{x_{j}^{2}-d_{1 n}^{2} / 2} \sqrt{v / \sigma_{n}^{2}}\right] w_{j} .
\end{aligned}
$$

Note that in this case we no longer have the equality $d_{1 n}=\sqrt{2} x_{j}$ as $v \neq \sigma_{n}^{2}$. One can appropriately regard $v$ as a weighted average of $\sigma_{n}^{2}$ for all $n$ 's. 


\section{References}

Ait-Sahalia, Yacine, 2002, Telling from discrete data whether the underlying continuous-time model is a diffusion, Journal of Finance October, forthcoming.

Bates, David, 1991, The crash of 87: Was it expected? The evidence from option markets, Journal of Finance 46, 1009-1044.

Bhattacharya, Mihir, 1980, Empirical properties of the blackscholes formula under ideal conditions, Journal of Financial and Quantitative Analysis 15, 1081-1105.

Black, Fisher, and Myron Scholes, 1972, The valuation of option contracts and a test of market efficiency, Journal of Finance 27, 399-417.

Bollen, Nick, and Emma Raisel, 2002, The performance of alternative valuation models in the otc currency option market, Journal of International Money and Finance forthcoming.

Boyle, P. P., and D. Emanuel, 1980, Discretely adjusted option hedges, Journal of Financial Economics 8, 259282.

Breeden, Douglas T., and Robert H. Litzenberger, 1978, Prices of state-contingent claims implicit in option prices, Journal of Business 51, 621-651.

Britten-Jones, Mark, and Anthony Neuberger, 2000, Option prices, implied price processes, and stochastic volatility, Journal of Finance 55, 839-866.

Carr, Peter, Katrina Ellis, and Vishal Gupta, 1998, Static hedging of exotic options, Journal of Finance 53, 1165-1190.

Carr, Peter, and Liuren Wu, 2001, A simple robust test for the presence of jumps in asset prices, Working paper, Fordham University.

Carr, Peter, and Liuren $\mathrm{Wu}, 2002$, Finite moment log stable process and option pricing, Journal of Finance forthcoming.

Cox, John C., and Stephen A. Ross, 1976, The valuation of options for alternative stochastic processes, Journal of Financial Economics 3, 145-166.

Davis, Philip J., and Philip Rabinowitz, 1984, Methods of Numerical Integration. (Academic Press New York).

Derman, Emanuel, and Michael Kamal, 1999, When you cannot hedge continuously, the corrections of blackscholes, Risk magazine. 
Dritschel, M., and Philip Protter, 1999, Complete markets with discontinuous security price, Finance and Stochastics 3, 203-214.

Dupire, Bruno, 1994, Pricing with a smile, Risk 7, 18-20.

Engle, Robert F., and Joshua V. Rosenberg, 1997, Empirical pricing kernels, manuscript, New York University New York.

Galai, D., 1983, The components of the return from hedging options against stocks, Journal of Business 56, $45-54$.

Green, Richard, and Robert A. Jarrow, 1987, Spanning and completeness in markets with contingent claims, Journal of Economic Theory 41, 202-210.

Heston, Stephen, 1993, Closed-form solution for options with stochastic volatility, with application to bond and currency options, Review of Financial Studies 6, 327-343.

Hodges, Stewart, and Anthony Neuberger, 2002, Rational bounds and the robust risk management of derivatives, manuscript, London Business School.

Jackwerth, Jens Carsten, and Mark Rubinstein, 1996, Recovering probability distributions from contemporary security prices, Journal of Finance 51, 347-369.

Leland, Hayne E., 1985, Option pricing and relation with transaction costs, Journal of Finance 40, 1283-1301.

Madan, Dilip, Peter Carr, and Eric Chang, 1998, The variance gamma process and option pricing, European Financial Review 2, 79-105.

Merton, Robert C., 1976, Option pricing when underlying stock returns are discontinuous, Journal of Financial Economics 3, 125-144.

Nachman, David, 1988, Spanning and completeness with options, Review of Financial Studies 3, 311-328.

Ross, Stephen A., 1976, Options and efficiency, Quarterly Journal of Economics 90, 75-89.

Toft, Klaus Bjerre, 1996, On the mean-variance tradeoff in option replication with transactions costs, Journal of Financial and Quantitative Analysis 31. 
Table I

Simulated Hedging Performance Comparisons of Static and Dynamic Strategies

Entries report the summary statistics (mean, standard error, root mean squared error (RMSE), mean absolute error (MAE), mean short fall (MSF), minimum, maximum, skewness, and kurtosis) of the hedging error of a one-year call option based on both static and dynamic strategies. The hedging error is defined as the difference between the value of the hedging portfolio and the value of the target call option being hedged at the closing of the month-long hedging exercise. The static hedging portfolios consist of several one-month call options with strikes and weights determined by the static relation and a Gauss-Hermite quadrature approximation. The portfolios are then hold for one month without rebalancing. The dynamic hedging portfolio is a simple delta hedging with the underlying futures, but with daily rebalancing. The statistics are computed based on 1,000 simulated paths of the Black-Scholes model (Panel A) and the Merton jump-diffusion model (Panel B), assuming that the hedger knows the exact model in forming the portflios. In Panel $\mathrm{C}$, the sample paths and option prices are simulated based on the Merton model, but we assume that the hedger does not know this information and is formed to form the hedging portfolios based on the Black-Scholes forumla, with ad hoc adjustments to accommodate the observed implied volatility. The last row of each panel reports the value of the target call option approximated by the quadrature method, with the theoretical value given under the dynamic hedging column. 


\begin{tabular}{|c|c|c|c|c|c|c|}
\hline \multirow{2}{*}{$\begin{array}{l}\text { Hedge Error } \\
\text { No. of Assets }\end{array}$} & \multicolumn{5}{|c|}{ Static with Options } & \multirow{2}{*}{$\begin{array}{c}\text { Dynamic with } \\
\text { Underlying }\end{array}$} \\
\hline & 3 & 5 & 10 & 15 & 21 & \\
\hline \multicolumn{7}{|c|}{ A. The Black-Scholes Model } \\
\hline Mean & -0.00 & 0.01 & -0.02 & 0.02 & 0.01 & 0.10 \\
\hline Std Err & 1.00 & 0.66 & 0.33 & 0.20 & 0.14 & 0.10 \\
\hline RMSE & 1.00 & 0.66 & 0.33 & 0.20 & 0.14 & 0.14 \\
\hline MAE & 0.86 & 0.57 & 0.29 & 0.18 & 0.13 & 0.12 \\
\hline MSF & -0.43 & -0.28 & -0.15 & -0.08 & -0.06 & -0.01 \\
\hline Min & -1.62 & -1.13 & -0.58 & -0.38 & -0.25 & -0.43 \\
\hline Max & 1.86 & 0.93 & 0.40 & 0.24 & 0.17 & 0.32 \\
\hline Skewness & 0.01 & -0.26 & -0.32 & -0.61 & -0.48 & -0.84 \\
\hline Kurtosis & 1.87 & 1.79 & 1.70 & 1.96 & 1.78 & 4.68 \\
\hline Call Value & 11.72 & 12.20 & 12.34 & 12.37 & 12.36 & 12.35 \\
\hline \multicolumn{7}{|c|}{ B. The Merton Jump-Diffusion Model } \\
\hline Mean & -0.01 & 0.00 & -0.01 & 0.02 & 0.02 & 0.07 \\
\hline Std Err & 0.72 & 0.47 & 0.31 & 0.16 & 0.12 & 1.05 \\
\hline RMSE & 0.72 & 0.47 & 0.31 & 0.16 & 0.12 & 1.05 \\
\hline MAE & 0.53 & 0.35 & 0.27 & 0.13 & 0.10 & 0.48 \\
\hline MSF & -0.27 & -0.18 & -0.14 & -0.05 & -0.04 & -0.20 \\
\hline Min & -1.73 & -1.28 & -0.67 & -0.58 & -0.41 & -12.12 \\
\hline Max & 2.84 & 1.48 & 0.51 & 0.20 & 0.14 & 0.37 \\
\hline Skewness & 0.56 & -0.16 & -0.10 & -1.77 & -1.65 & -6.82 \\
\hline Kurtosis & 5.23 & 4.07 & 1.83 & 5.74 & 5.15 & 59.79 \\
\hline Call Value & 9.52 & 11.14 & 11.93 & 12.09 & 12.06 & 11.99 \\
\hline \multicolumn{7}{|c|}{ C. Ad Hoc Black-Scholes Hedge under the Merton World } \\
\hline Mean & -0.00 & 0.01 & -0.02 & 0.01 & 0.01 & 0.03 \\
\hline Std Err & 0.61 & 0.38 & 0.46 & 0.19 & 0.21 & 1.04 \\
\hline RMSE & 0.61 & 0.38 & 0.46 & 0.19 & 0.21 & 1.04 \\
\hline MAE & 0.48 & 0.29 & 0.39 & 0.12 & 0.15 & 0.45 \\
\hline MSF & -0.24 & -0.14 & -0.21 & -0.05 & -0.07 & -0.21 \\
\hline Min & -1.57 & -1.30 & -0.72 & -0.92 & -0.82 & -12.48 \\
\hline Max & 1.63 & 1.10 & 0.85 & 0.68 & 0.65 & 2.08 \\
\hline Skewness & -0.26 & -0.43 & 0.12 & -0.43 & -0.61 & -6.45 \\
\hline Kurtosis & 3.13 & 4.07 & 1.87 & 9.23 & 5.61 & 56.05 \\
\hline Call Value & 11.53 & 11.96 & 11.84 & 12.07 & 12.03 & 11.99 \\
\hline
\end{tabular}


Table II

Effects of Rebalancing Frequencies on the Dynamic Delta Hedge

Entries report the summary statistics (mean, standard error, root mean squared error (RMSE), mean absolute error (MAE), mean short fall (MSF), minimum, maximum, skewness, and kurtosis) of the hedging error of a one-year call option based on a dynamic delta hedge with different rebalancing frequencies. The hedging error is defined as the difference between the value of the hedging portfolio and the value of the target call option at the closing time of the month-long exercise. The statistics are computed based on 1,000 simulated paths of the Black-Scholes model (Panel A) and the Merton jump-diffusion model (Panel B)assuming that the hedger knows the exact model in forming the portflios. In Panel $\mathrm{C}$, the sample paths and option prices are simulated based on the Merton model, but we assume that the hedger does not know this information and is formed to form the hedging portfolios based on the Black-Scholes forumla, with ad hoc adjustments to accommodate the observed implied volatility.

\begin{tabular}{|c|c|c|c|c|}
\hline \multirow[t]{2}{*}{ Statistics } & \multicolumn{4}{|c|}{ Reblancing Frequency Per Day } \\
\hline & 1 & 2 & 5 & 10 \\
\hline \multicolumn{5}{|c|}{ A. The Black-Scholes Model } \\
\hline Mean & 0.11 & 0.11 & 0.11 & 0.11 \\
\hline Std Err & 0.10 & 0.07 & 0.04 & 0.03 \\
\hline RMSE & 0.15 & 0.13 & 0.12 & 0.12 \\
\hline MAE & 0.13 & 0.12 & 0.11 & 0.11 \\
\hline MSF & -0.01 & -0.00 & -0.00 & 0.00 \\
\hline Min & -0.36 & -0.15 & -0.03 & 0.02 \\
\hline Max & 0.32 & 0.28 & 0.22 & 0.19 \\
\hline Skewness & -0.77 & -0.41 & -0.34 & -0.16 \\
\hline Kurtosis & 4.21 & 3.23 & 3.12 & 2.86 \\
\hline
\end{tabular}

B. The Merton Jump-Diffusion Model

$\begin{array}{lrrrr}\text { Mean } & 0.09 & 0.09 & 0.09 & 0.09 \\ \text { Std Err } & 1.02 & 1.03 & 1.03 & 1.02 \\ \text { RMSE } & 1.02 & 1.03 & 1.03 & 1.03 \\ \text { MAE } & 0.49 & 0.49 & 0.49 & 0.49 \\ \text { MSF } & -0.20 & -0.20 & -0.20 & -0.20 \\ \text { Min } & -11.78 & -11.84 & -11.72 & -11.72 \\ \text { Max } & 0.38 & 0.37 & 0.35 & 0.35 \\ \text { Skewness } & -6.27 & -6.30 & -6.24 & -6.20 \\ \text { Kurtosis } & 50.97 & 51.40 & 50.34 & 49.79\end{array}$

\section{Ad Hoc Black-Scholes Hedge under the Merton World}

$\begin{array}{lrrrr}\text { Mean } & 0.08 & 0.09 & 0.09 & 0.07 \\ \text { Std Err } & 0.88 & 0.92 & 0.93 & 0.88 \\ \text { RMSE } & 0.88 & 0.93 & 0.93 & 0.88 \\ \text { MAE } & 0.41 & 0.41 & 0.42 & 0.41 \\ \text { MSF } & -0.17 & -0.16 & -0.16 & -0.17 \\ \text { Min } & -10.13 & -10.19 & -10.08 & -10.07 \\ \text { Max } & 1.21 & 8.97 & 9.43 & 0.76 \\ \text { Skewness } & -6.31 & -4.74 & -4.43 & -6.25 \\ \text { Kurtosis } & 52.66 & 54.10 & 53.41 & 51.59\end{array}$


Table III

Effects of Target and Hedging Instrument Choice

Entries report the summary statistics (mean, standard error, root mean squared error (RMSE), mean absolute error (MAE), mean short fall (MSF), minimum, maximum, skewness, and kurtosis) of the hedging errors when hedging different target options and using different hedging hedging instruments. The first row denotes the maturity of the target at-the-money option being hedged. The second row denotes the strategy, and the third row denotes the maturity of the options in the case of the static hedging strategy. The statistics are computed based on 1,000 simulated paths of the Black-Scholes model (Panel A) and the Merton jump-diffusion model (Panel B)assuming that the hedger knows the exact model in forming the portflios. In Panel $\mathrm{C}$, the sample paths and option prices are simulated based on the Merton model, but we assume that the hedger does not know this information and is formed to form the hedging portfolios based on the Black-Scholes forumla, with ad hoc adjustments to accommodate the observed implied volatility. The last row of each panel reports the value of the target call option approximated by the quadrature method, with the theoretical value given under the dynamic hedging column. 


\begin{tabular}{|c|c|c|c|c|c|c|c|c|c|c|c|c|c|}
\hline Target Mat & 2 & 4 & 12 & 12 & 12 & 2 & 4 & 12 & 12 & 12 & 2 & 4 & 12 \\
\hline Strategy & \multicolumn{5}{|c|}{ Static with Three Options } & \multicolumn{5}{|c|}{ Static with Five Options } & & De & \\
\hline Instruments & 1 & 1 & 1 & 2 & 4 & 1 & 1 & 1 & 2 & 4 & Unde & $\lg \mathrm{F}$ & res \\
\hline
\end{tabular}

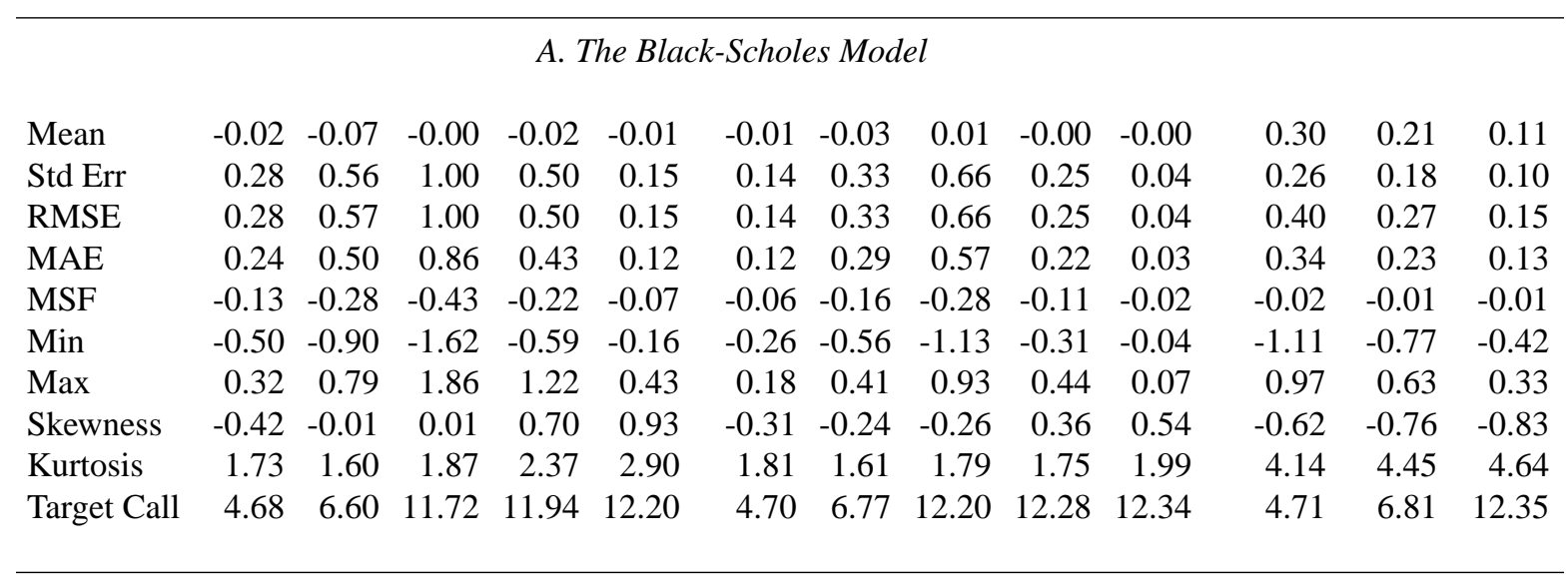

\section{B. The Merton Jump-Diffusion Model}

\begin{tabular}{lrrrrrrrrrrrrr} 
Mean & -0.10 & -0.09 & -0.01 & -0.02 & -0.02 & -0.06 & -0.05 & 0.00 & -0.00 & -0.01 & 0.23 & 0.16 & 0.09 \\
Std Err & 0.84 & 0.77 & 0.72 & 0.50 & 0.27 & 0.40 & 0.46 & 0.47 & 0.29 & 0.16 & 2.55 & 1.92 & 1.05 \\
RMSE & 0.85 & 0.77 & 0.72 & 0.50 & 0.27 & 0.40 & 0.46 & 0.47 & 0.29 & 0.16 & 2.56 & 1.93 & 1.05 \\
MAE & 0.68 & 0.64 & 0.53 & 0.35 & 0.14 & 0.34 & 0.37 & 0.35 & 0.21 & 0.06 & 1.33 & 0.95 & 0.49 \\
MSF & -0.39 & -0.36 & -0.27 & -0.19 & -0.08 & -0.20 & -0.21 & -0.18 & -0.11 & -0.03 & -0.55 & -0.40 & -0.20 \\
Min & -1.20 & -1.20 & -1.73 & -0.81 & -0.33 & -0.69 & -0.74 & -1.28 & -0.72 & -0.64 & -25.22 & -19.83 & -12.11 \\
Max & 6.08 & 2.76 & 2.84 & 2.49 & 1.95 & 1.87 & 1.26 & 1.48 & 1.25 & 0.98 & 1.14 & 0.76 & 0.38 \\
Skewness & 1.29 & 0.51 & 0.56 & 1.76 & 4.76 & 0.11 & 0.66 & -0.16 & 1.13 & 4.44 & -5.51 & -5.85 & -6.81 \\
Kurtosis & 7.89 & 2.65 & 5.23 & 9.43 & 29.43 & 2.67 & 3.38 & 4.07 & 7.10 & 26.23 & 39.24 & 43.87 & 59.66 \\
Target Call & 3.76 & 4.80 & 9.52 & 9.76 & 10.15 & 3.72 & 5.33 & 11.14 & 11.19 & 11.18 & 4.11 & 6.34 & 11.99 \\
& & & & & & & & & & & & \\
\hline
\end{tabular}

\section{Ad Hoc Black-Scholes Hedge under the Merton World}

\begin{tabular}{lrrrrrrrrrrrrr} 
Mean & -0.10 & -0.07 & -0.01 & -0.02 & -0.02 & -0.06 & -0.04 & 0.00 & -0.00 & -0.00 & 0.18 & 0.11 & 0.05 \\
Std Err & 0.84 & 0.55 & 0.63 & 0.46 & 0.27 & 0.40 & 0.31 & 0.41 & 0.26 & 0.14 & 2.26 & 1.73 & 1.04 \\
RMSE & 0.85 & 0.55 & 0.63 & 0.46 & 0.27 & 0.40 & 0.31 & 0.41 & 0.26 & 0.14 & 2.26 & 1.73 & 1.05 \\
MAE & 0.68 & 0.43 & 0.45 & 0.31 & 0.11 & 0.34 & 0.25 & 0.32 & 0.19 & 0.06 & 1.18 & 0.85 & 0.47 \\
MSF & -0.39 & -0.25 & -0.23 & -0.17 & -0.06 & -0.20 & -0.14 & -0.16 & -0.10 & -0.03 & -0.50 & -0.37 & -0.21 \\
Min & -1.20 & -0.81 & -1.42 & -0.70 & -0.65 & -0.69 & -0.53 & -1.10 & -0.67 & -0.59 & -22.01 & -17.43 & -12.46 \\
Max & 6.08 & 1.59 & 2.53 & 2.38 & 1.87 & 1.87 & 0.89 & 1.12 & 1.07 & 0.83 & 2.36 & 2.16 & 2.10 \\
Skewness & 1.29 & 1.06 & 0.81 & 2.16 & 5.12 & 0.11 & 0.47 & -0.42 & 0.92 & 4.13 & -5.38 & -5.68 & -6.45 \\
Kurtosis & 7.89 & 4.26 & 5.93 & 11.28 & 31.09 & 2.67 & 3.25 & 3.53 & 6.27 & 24.24 & 38.74 & 42.88 & 55.98 \\
Target Call & 3.76 & 4.94 & 10.03 & 9.94 & 10.24 & 3.72 & 5.80 & 11.55 & 11.38 & 11.34 & 4.11 & 6.34 & 11.99 \\
& & & & & & & & & & & & & \\
\hline \hline
\end{tabular}




\section{Table IV \\ Mean Absolute Hedging Errors on S\&P 500 Index Options}

Entries report the mean absolute hedging errors at each weekday of the one-month hedging horizon. The maturities (in months) of options being hedged are given in the first row. They are near-the-money options. The hedging strategy is either static with a portfolio of three options, five options, or dynamic with the underlying futures and daily updating. The maturity of the options in the static hedging portfolio (in months) are given in the third row. The first two columns of the table denote the week, and weekdays on which the hedging errors are recorded.

\begin{tabular}{|c|c|c|c|c|c|c|c|c|c|c|c|c|c|c|}
\hline \multicolumn{2}{|c|}{ Option Maturity } & 2 & $4-6$ & 12 & 12 & 12 & 2 & $4-6$ & 12 & 12 & 12 & 2 & $4-6$ & 12 \\
\hline \multirow{2}{*}{\multicolumn{2}{|c|}{$\begin{array}{l}\text { Strategy } \\
\text { Instruments }\end{array}$}} & \multicolumn{5}{|c|}{ Static with Three Options } & \multicolumn{5}{|c|}{ Static with Five Options } & \multirow{2}{*}{\multicolumn{3}{|c|}{$\begin{array}{c}\text { Daily Delta } \\
\text { Underlying Futures }\end{array}$}} \\
\hline & & 1 & 1 & 1 & 2 & $4-6$ & 1 & 1 & 1 & 2 & $4-6$ & & & \\
\hline \multirow[t]{3}{*}{ I } & Wed & 0.00 & 0.00 & 0.00 & 0.00 & 0.00 & 0.00 & 0.00 & 0.00 & 0.00 & 0.00 & 0.00 & 0.00 & 0.00 \\
\hline & Thu & 0.59 & 0.77 & 2.18 & 1.67 & 1.33 & 0.57 & 0.62 & 1.80 & 1.42 & 1.24 & 1.71 & 1.50 & 2.21 \\
\hline & Fri & 0.64 & 1.16 & 2.65 & 2.00 & 1.44 & 0.69 & 1.04 & 2.18 & 1.68 & 1.39 & 2.00 & 2.10 & 2.56 \\
\hline \multirow[t]{5}{*}{ II } & Mon & 0.73 & 1.57 & 3.46 & 2.67 & 1.80 & 0.76 & 1.38 & 2.85 & 2.23 & 1.67 & 2.11 & 2.16 & 2.88 \\
\hline & Tue & 0.81 & 1.64 & 3.98 & 2.82 & 2.00 & 0.86 & 1.47 & 3.24 & 2.38 & 1.98 & 2.28 & 2.46 & 3.38 \\
\hline & Wed & 0.90 & 1.89 & 4.20 & 3.12 & 2.24 & 0.93 & 1.69 & 3.57 & 2.74 & 2.31 & 2.62 & 2.78 & 3.89 \\
\hline & Thu & 1.02 & 1.79 & 4.39 & 3.19 & 2.25 & 1.13 & 1.56 & 3.64 & 2.75 & 2.23 & 2.83 & 2.91 & 3.97 \\
\hline & Fri & 1.10 & 2.28 & 5.31 & 3.68 & 2.49 & 1.17 & 2.04 & 4.32 & 3.07 & 2.47 & 3.21 & 3.40 & 4.15 \\
\hline \multirow[t]{5}{*}{ III } & Mon & 1.18 & 2.34 & 4.75 & 3.37 & 2.18 & 1.22 & 2.14 & 3.99 & 2.89 & 2.22 & 3.35 & 3.55 & 4.95 \\
\hline & Tue & 1.19 & 2.56 & 5.72 & 4.13 & 2.60 & 1.25 & 2.20 & 4.79 & 3.58 & 2.58 & 3.26 & 3.54 & 4.77 \\
\hline & Wed & 1.26 & 2.78 & 6.32 & 4.31 & 2.54 & 1.31 & 2.36 & 5.12 & 3.56 & 2.51 & 3.51 & 3.56 & 4.77 \\
\hline & Thu & 1.32 & 3.16 & 7.62 & 5.68 & 3.72 & 1.43 & 2.75 & 6.52 & 4.92 & 3.55 & 3.42 & 3.87 & 6.04 \\
\hline & Fri & 1.51 & 3.12 & 8.12 & 5.89 & 3.88 & 1.49 & 2.70 & 6.89 & 5.16 & 3.68 & 3.78 & 4.20 & 6.43 \\
\hline \multirow[t]{5}{*}{ IV } & Mon & 1.46 & 2.97 & 7.74 & 5.70 & 3.87 & 1.58 & 2.54 & 5.84 & 4.28 & 2.88 & 3.84 & 3.83 & 5.85 \\
\hline & Tue & 1.55 & 3.28 & 8.42 & 6.09 & 4.02 & 1.68 & 2.83 & 6.33 & 4.69 & 3.04 & 4.06 & 3.98 & 5.82 \\
\hline & Wed & 1.50 & 3.64 & 8.84 & 6.10 & 3.95 & 1.62 & 3.05 & 6.50 & 4.64 & 3.03 & 4.27 & 4.26 & 5.77 \\
\hline & Thu & 1.55 & 3.60 & 9.51 & 6.65 & 4.30 & 1.71 & 2.95 & 7.41 & 5.17 & 3.42 & 4.24 & 4.17 & 6.27 \\
\hline & Fri & 1.77 & 4.31 & 10.19 & 6.71 & 4.09 & 1.88 & 3.49 & 7.56 & 5.04 & 3.26 & 4.56 & 4.31 & 6.23 \\
\hline \multirow[t]{4}{*}{ V } & Mon & 1.87 & 4.73 & 10.47 & 6.98 & 4.28 & 1.89 & 3.84 & 7.85 & 5.36 & 3.45 & 4.81 & 4.84 & 6.64 \\
\hline & Tue & 1.77 & 4.93 & 10.39 & 6.90 & 4.37 & 1.89 & 3.79 & 7.82 & 5.38 & 3.62 & 4.64 & 4.58 & 6.32 \\
\hline & Wed & 1.99 & 5.13 & 10.22 & 6.98 & 4.42 & 1.97 & 3.93 & 7.65 & 5.32 & 3.52 & 4.92 & 4.92 & 7.02 \\
\hline & Thu & 2.42 & 5.73 & 10.85 & 7.42 & 4.61 & 2.08 & 4.35 & 8.20 & 5.74 & 3.74 & 5.38 & 5.38 & 7.45 \\
\hline
\end{tabular}


Table V

\section{Locations of Large Absolute Hedge Errors}

Entries report the month that generates the largest absolute hedging error at each week day during the month-long hedging exercise. The first row reports the maturity of the option being hedged. The second row states the hedging strategy. The starting date of each month is listed in Table VI.

\begin{tabular}{|c|c|c|c|c|c|c|c|}
\hline \multicolumn{2}{|c|}{ Option Maturity } & 2 & 4-6 & 12 & 2 & $4-6$ & 12 \\
\hline \multicolumn{2}{|c|}{ Strategy } & \multicolumn{3}{|c|}{ Daily Delta with Futures } & \multicolumn{3}{|c|}{ Static with $51 \mathrm{M}$ Options } \\
\hline \multirow[t]{5}{*}{ II } & Mon & 22 & 22 & 45 & 51 & 51 & 38 \\
\hline & Tue & 22 & 22 & 31 & 51 & 33 & 38 \\
\hline & Wed & 22 & 22 & 31 & 69 & 33 & 33 \\
\hline & Thu & 22 & 22 & 38 & 32 & 33 & 43 \\
\hline & Fri & 22 & 22 & 22 & 32 & 32 & 32 \\
\hline \multirow[t]{5}{*}{ III } & Mon & 22 & 22 & 31 & 32 & 22 & 32 \\
\hline & Tue & 22 & 22 & 31 & 27 & 51 & 32 \\
\hline & Wed & 22 & 22 & 22 & 27 & 33 & 32 \\
\hline & Thu & 22 & 22 & 72 & 27 & 33 & 72 \\
\hline & Fri & 22 & 22 & 72 & 27 & 42 & 72 \\
\hline \multirow[t]{5}{*}{ IV } & Mon & 22 & 22 & 72 & 27 & 33 & 33 \\
\hline & Tue & 22 & 22 & 72 & 36 & 33 & 33 \\
\hline & Wed & 22 & 22 & 72 & 27 & 33 & 33 \\
\hline & Thu & 22 & 22 & 72 & 43 & 33 & 33 \\
\hline & Fri & 51 & 22 & 72 & 43 & 33 & 33 \\
\hline \multirow[t]{4}{*}{$\mathrm{V}$} & Mon & 22 & 22 & 72 & 43 & 33 & 33 \\
\hline & Tue & 22 & 22 & 72 & 43 & 33 & 33 \\
\hline & Wed & 61 & 22 & 72 & 43 & 33 & 33 \\
\hline & Thu & 61 & 22 & 72 & 36 & 33 & 32 \\
\hline
\end{tabular}


Table VI

Months With Large Absolute Hedge Errors

Entries report the starting dates of the months that generate the large absolute hedging errors. The exact dates at which these months generate large absolute hedging errors are listed in Table V.

\begin{tabular}{llllll}
\hline \hline Number & Starting Dates & Number & Starting Dates & Number & Starting Dates \\
\hline 22 & $10 / 22 / 1997$ & 36 & $12 / 16 / 1998$ & 51 & $03 / 22 / 2000$ \\
27 & $03 / 18 / 1998$ & 38 & $02 / 17 / 1999$ & 61 & $01 / 17 / 2001$ \\
31 & $07 / 22 / 1998$ & 42 & $06 / 16 / 1999$ & 69 & $09 / 19 / 2001$ \\
32 & $08 / 19 / 1998$ & 43 & $07 / 21 / 1999$ & 72 & $12 / 19 / 2001$ \\
33 & $09 / 16 / 1998$ & 45 & $09 / 15 / 1999$ & & \\
\hline \hline
\end{tabular}

